\title{
Multicellular Rosettes Drive Fluid-solid Transition in Epithelial Tissues
}

\author{
Le Yan* \\ Kavli Institute for Theoretical Physics, University of California, Santa Barbara, California 93106, USA \\ Dapeng $\mathrm{Bi}^{\dagger}$ \\ Department of Physics, Northeastern University, Massachusetts 02115, USA
}

(Received 7 June 2018; revised manuscript received 12 November 2018; published 12 February 2019)

\begin{abstract}
Models for confluent biological tissues often describe the network formed by cells as a triplejunction network, similar to foams. However, higher-order vertices or multicellular rosettes are prevalent in developmental and in vitro processes and have been recognized as crucial in many important aspects of morphogenesis, disease, and physiology. In this work, we study the influence of rosettes on the mechanics of a confluent tissue. We find that the existence of rosettes in a tissue can greatly influence its rigidity. Using a generalized vertex model and the effective medium theory, we find a fluid-to-solid transition driven by rosette density and intracellular tensions. This transition exhibits several hallmarks of a second-order phase transition such as a growing correlation length and a universal critical scaling in the vicinity of a critical point. Furthermore, we elucidate the nature of rigidity transitions in dense biological tissues and other cellular structures using a generalized Maxwell constraint counting approach, which answers a long-standing puzzle of the origin of solidity in these systems.
\end{abstract}

DOI: 10.1103/PhysRevX.9.011029

Subject Areas: Biological Physics, Soft Matter

\section{INTRODUCTION}

Multicellular organization in tissues is important to understanding many aspects of biology and medicine, such as embryonic development, disease generation, and progression. Particularly in fully confluent epithelial tissues, where cells are densely packed and have tight adherent junctions between them, the behavior and response of cells can be highly collective and differ from the single-cell-level behavior. Many models have been proposed to understand the emergence of this collective behavior and the biophysical properties of tissues. In the past two decades, a class of cell-based models known as the vertex model has been developed to study tissue mechanics [1-25]. In the vertex model, each cell is represented as a deformable polygonal inclusion, with edges and vertices shared by neighboring cells. This class of models often assumes that exactly three cells meet at any vertex in an epithelial tissue and fourfold vertices occur only as an intermediate state during a $T 1$ rearrangement.

\footnotetext{
*lyan@kitp.ucsb.edu

†d.bi@northeastern.edu
}

Published by the American Physical Society under the terms of the Creative Commons Attribution 4.0 International license. Further distribution of this work must maintain attribution to the author(s) and the published article's title, journal citation, and DOI.
However, in many tissues [26], higher-order vertices where four or more cells meet can occur. A prominent example occurs in Drosophila embryogenesis, where a mixture of $T 1$ junctions [27-31] (vertices where four cells meet) and multicellular rosettes [32-37] (vertices with five or more cells) have been observed during the elongation of the body axis. It has been further shown that, as the body length of the embryo doubles due to a series of cellular rearrangements, a majority of cells in the developing epithelium participate in rosette formation $[33,36]$. The morphogenesis of the Drosophila eye is also facilitated by rosettes [38]. In zebrafish lateral-line development, the lateral line is composed of mechanosensory organs called neuromasts, which are formed from rosettes composed of 20 or more cells [39-41]. In other vertebrates, rosettes have been observed in the neural plate of chick [42] and mouse [43] embryos, in the development of the mouse visceral endoderm [44], the kidney tubule [45], and the pancreas [46]. They are also observed in adults and in vitro such as adult mammalian brains and cultured neural stem cells $[47,48]$.

The importance of cellular rosettes has been widely recognized. They have been proposed as an efficient mechanism for tissue remodeling and growth during the body elongation in the Drosophila embryo $[33,35,36]$ and are thought to be crucial for the orderliness of collective cell migration in the mouse visceral endoderm [26,44]. Cancer pathologists visually inspecting histologic samples from tumors use cellular rosettes as strong indications of 
malignancy such as medulloblastoma and retinoblastoma [49]. However, compared to the large body of live-imaging and molecular studies [26], there are surprisingly few modeling [44] and even less physical understanding of rosettes regarding how they influence tissue mechanics.

Here, we develop a generalized vertex model and an effective medium theory that takes into account the presence of rosettes. We also make experimentally testable predictions regarding the strong correlations and the interplay between cellular topology and mechanical tensions in a tissue. We find that the tissue rigidity is precisely controlled by the density of higher-order vertices including rosettes and $T 1$ junctions as well as a single parameter that tunes intracellular tensions in the tissue. The results show that a tissue behaving as a fluid can be rigidified by the creation of just a few higher-order vertices. The transition between fluid and rigid states exhibit many hallmarks of a second-order phase transition, such as a growing correlation length and a universal critical scaling in the vicinity of a critical point. Furthermore, we offer a unifying perspective on rigidity transitions in dense confluent tissues and cellular materials by elucidating the nature of this transition using a generalized Maxwell constraint counting.

\section{GENERALIZED VERTEX MODEL}

We begin with the most generic form of the vertex model for a homogeneous tissue [2], where the total energy is given by a sum over the mechanical cost of deforming individual cells:

$$
U=\sum_{\alpha=1}^{F}\left[K_{A}\left(A_{\alpha}-A_{0}\right)^{2}+K_{P}\left(P_{\alpha}-P_{0}\right)^{2}\right] .
$$

The first term results from a combination of threedimensional cell incompressibility and the monolayer's resistance to height fluctuations or cell bulk elasticity $[3,8]$, where $K_{A}$ is a height elasticity, $A_{\alpha}$ is the cross-sectional area (apical) of cell $\alpha$, and $A_{0}$ is the preferred area for the cell. The second term in Eq. (1) is quadratic in the cell cross-sectional perimeter $P_{\alpha}$ and models the active contractility of the actin-myosin subcellular cortex, with elastic constant $K_{P}$ [2], and $P_{0}$ is an effective target shape index, representing an interfacial tension set by a competition between the cortical tension and the energy of cell-cell adhesion $[5,8]$ between two contacting cells. In Eq. (1), the sum is over all $F$ number of cells in the tissue. The cell areas $\left(\left\{A_{\alpha}\right\}\right)$ and perimeter $\left(\left\{P_{\alpha}\right\}\right)$ are fully determined by the positions of vertices $\left\{\mathbf{R}_{i}\right\}$. Because of a combination of cortical tension and cell-cell adhesion [2,50], each cell contributes an effective line tension [10] to adjacent edges, controlled by $P_{0}$, which can also be interpreted as a geometrical nondimensional shape index $p_{0}=P_{0} / \sqrt{A_{0}}$. It is demonstrated that $[2,8,18]$ the tissue undergoes a phase transition at $p_{0}=p_{0}^{*} \approx 3.81$, independent of the strength of area constraints $\left(K_{A} A_{0}\right) /\left(K_{P}\right)$. When $p_{0} \leq p_{0}^{*}$, the tissue behaves like a rigid solid with a finite shear modulus. Above $p_{0}^{*}$, the tissue becomes soft and fluidlike with a vanishing shear modulus.

\section{MANIPULATION OF CELLULAR TOPOLOGY AND THE CREATION OF HIGHER-ORDER VERTICES}

In developmental and in vitro examples, the general mechanism for rosette formation is the contraction of actomyosin networks in cells [26]. This mechanism can be manifested in several ways. For example, in Drosophila body-axis elongation, rosettes are due to planar polarized constriction $[28,29,31,33,34,36]$, while in gastrulation and neural tube closure, actomyosin structures in the apical cell surface constrict to form rosettes [39-41]. Also, when cells delaminate or extrude from epithelia [51-54], a vertex with more than four edges can be left behind that becomes a center of a rosette. Similarly, a multicellular rosette can also form as the result of a wound closure [55,56].

Here, instead of focusing on the origin of cellular rosettes which can be varied for different processes, we assume that they have been created via one of the observed mechanisms and ask how their presence affects the mechanics at the tissue level. In practice, we create rosettes and $T 1$ junctions via a simple protocol of the random collapse of edges. During this process, an edge is chosen at random, and its length is reduced to zero. The two vertices on the end of the edge are then merged into a single vertex. The fractions of $T 1$ vertices and rosettes generated in this random protocol turn out to be consistent with the fractions in the fly embryo epithelial tissue during the germ band extension [33]. We carry out this process while making sure the number of cells $F$ remains constant. These moves model the convergence of vertices in developmental processes and cell-extrusion events.

Under periodic boundary conditions, the network will always obey Euler's characteristic formula $V-E+F=0$, where $V$ and $E$ are the number of vertices and edges, respectively. The density of higher-order vertices is captured by the average vertex coordination number, given by

$$
Z=2 E / V \text {. }
$$

Beginning with a tissue at $Z=3$ (i.e., containing only trijunctions), we choose an arbitrary value of $Z$ between 3 and 6 by applying a series of edge-collapse moves. Representative simulation snapshots are shown in Fig. 1. After $Z$ is changed, the energy is minimized using the conjugate-gradient method. For simplicity, no additional topological changes are performed (e.g., $T 1$ transitions, cell divisions, or cell apoptosis or extrusion) during the minimization, which corresponds to looking at when higherorder vertices are formed but not immediately resolved $[31,33-36,57]$ or in systems where they are persistent for 


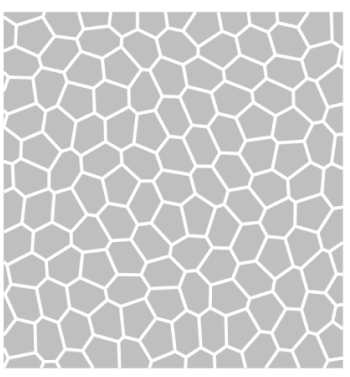

$Z=3$

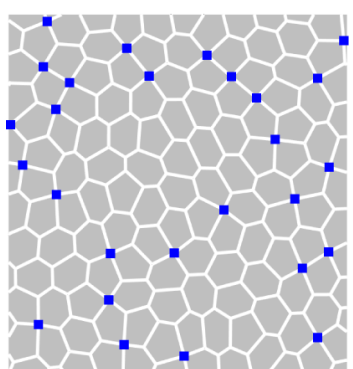

$Z=3.14$

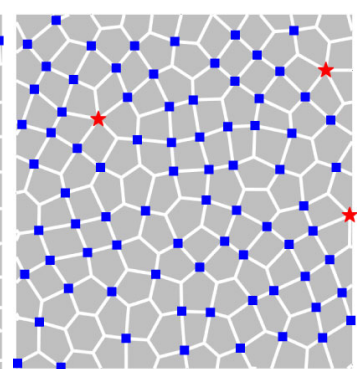

$Z=3.57$

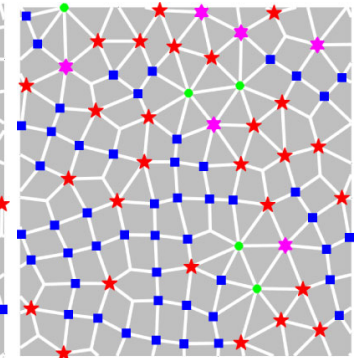

$Z=4.25$

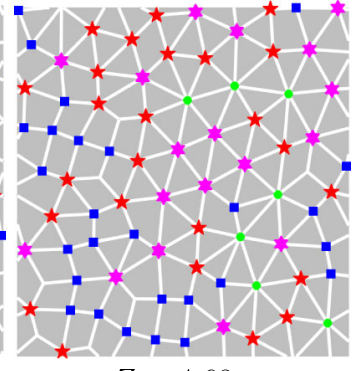

$Z=4.68$

FIG. 1. Introducing higher-order vertices into the vertex model. In conventional vertex models, all vertices have threefold coordination. Here, we apply a protocol that randomly collapses cell edges to create vertices with coordination higher than 3 . Fourfold vertices are called "T1 junctions," and fivefold or higher vertices are termed rosettes. We characterize the state of a tissue by the average vertex coordination number $Z$. Simulation snapshots show states with different values of $Z$. Squares, five-pointed stars, and six-pointed stars indicate vertices with a coordination of 4,5 , or 6 , respectively. Disks correspond to vertices with a coordination of 7 or more.

extended periods of time $[43,44,58,59]$. We also perform an alternative set of simulations in which $T 1$ and $T 2$ (cell apoptosis) transitions are allowed as shown in Fig. 11 which do not affect our findings. We apply the protocol using two types of initial states at $Z=3$ : (i) random tissue networks, where cell shapes are obtained from a random Voronoi tessellation $[9,24]$, and (ii) an ordered hexagonal tiling $[2,4]$, where every cell is a regular hexagon.

\section{TISSUE MECHANICAL RIGIDITY TRANSITIONS}

We begin by probing the mechanical response of the tissue as a function of the average coordination number $Z$ and the nondimensional cell shape index $p_{0}$. In order to determine whether the tissue is mechanically rigid, we analyze the zero modes of the Hessian matrix

$$
M_{i \mu j \nu}=\frac{\partial^{2} U}{\partial R_{i \mu} \partial R_{j \nu}}
$$

where $\mathbf{R}_{i}$ and $\mathbf{R}_{j}$ are positions of vertices $i$ and $j$, respectively, while $\mu$ and $\nu$ are Cartesian coordinates. The Hessian has dimensions of $2 V \times 2 V$ and a eigenspectrum of $2 V$ eigenvalues $\left\{\lambda_{k}=\omega_{k}^{2}\right\}$. The presence of zero eigenvalues indicates the loss of rigidity, which would correspond to floppy modes or zero modes that allow deformation of the system without changes in the total energy. The number of floppy modes $N_{0}$ can therefore be used as a measure to distinguish between the rigid and fluid states. Mathematically, the number of floppy modes is just the nullity of $M$, i.e., number of linearly independent solutions to $M|\delta \mathbf{R}\rangle=\mathbf{0}$.

In Fig. 2(a), the rigid states $\left(N_{0}=0\right)$ and the fluid states $\left(N_{0}>0\right)$ are shown for simulation results at all value pairs $\left(p_{0}, Z\right)$. At $Z=3$, the tissue undergoes a rigidity transition at $p_{0}=p_{0}^{*} \approx 3.818 \quad\left[p_{0}^{*} \approx 3.722\right.$ for the hexagonally ordered initial state (Fig. 9)]. This result recapitulates previous results on trijunction-only tissues $[2,4,8]$.
However, Fig. 2(a) further shows that a fluidized tissue at $Z=3$ can be rigidified when $Z$ is increased. The value of $Z$ where the tissue rigidifies depends on $p_{0}$. The boundary between rigid and nonrigid states follows a line of transition points $Z_{\text {crit }}\left(p_{0}\right)$ given by

$$
Z_{\text {crit }}\left(p_{0}\right)= \begin{cases}3 & p_{0} \leq p_{0}^{*}, \\ 3+\mathcal{B}\left[p_{0}-p_{0}^{*}\right] & p_{0}>p_{0}^{*}\end{cases}
$$

where the slope of the boundary separating solid and fluid states $(\mathcal{B} \approx 3.85)$ is obtained through empirical fitting. In Appendix B, we develop a mean-field model which provides an accurate prediction for the numerical value of $\mathcal{B}$.

To better understand how the creation of higher-order vertices can rigidify a tissue, we analyze the behavior of the fraction of zero modes $f_{0} \equiv N_{0} /(2 \mathrm{~V})$ as a function of $Z$ for various $p_{0}>p_{0}^{*}$ in Fig. 2(b). Using the relation of $Z_{\text {crit }}\left(p_{0}\right)$ [Eq. (4)], we then replot $f(\lambda=0)$ vs $Z-Z_{\text {crit }}$ in the inset in Fig. 2(b) and show that all curves can be collapsed onto a universal master curve. This result suggests that the nature of the rosette-driven rigidity transition may be universal across different $p_{0}$ values. Strikingly, $f_{0}$ decreases faster than the increasing number of topological constraints $Z$, which betrays the Maxwell counting theorem [60] in traditional rigidity transitions.

\section{A. Nature of the transition}

To explain the nature of the transition between the fluid and rigid states in Fig. 2(a), we employ a generalized version of the Maxwell constraint counting theorem [60]: The rigidity is lost when the number of independent constraints no longer matches the number of degrees of freedom (d.o.f.) ( $2 V$ for the model), and the number of floppy modes is given by the difference. It is tempting to simply prescribe one constraint per cell for each area term and each perimeter term in Eq. (1). However, this approach results in an erroneous counting of 

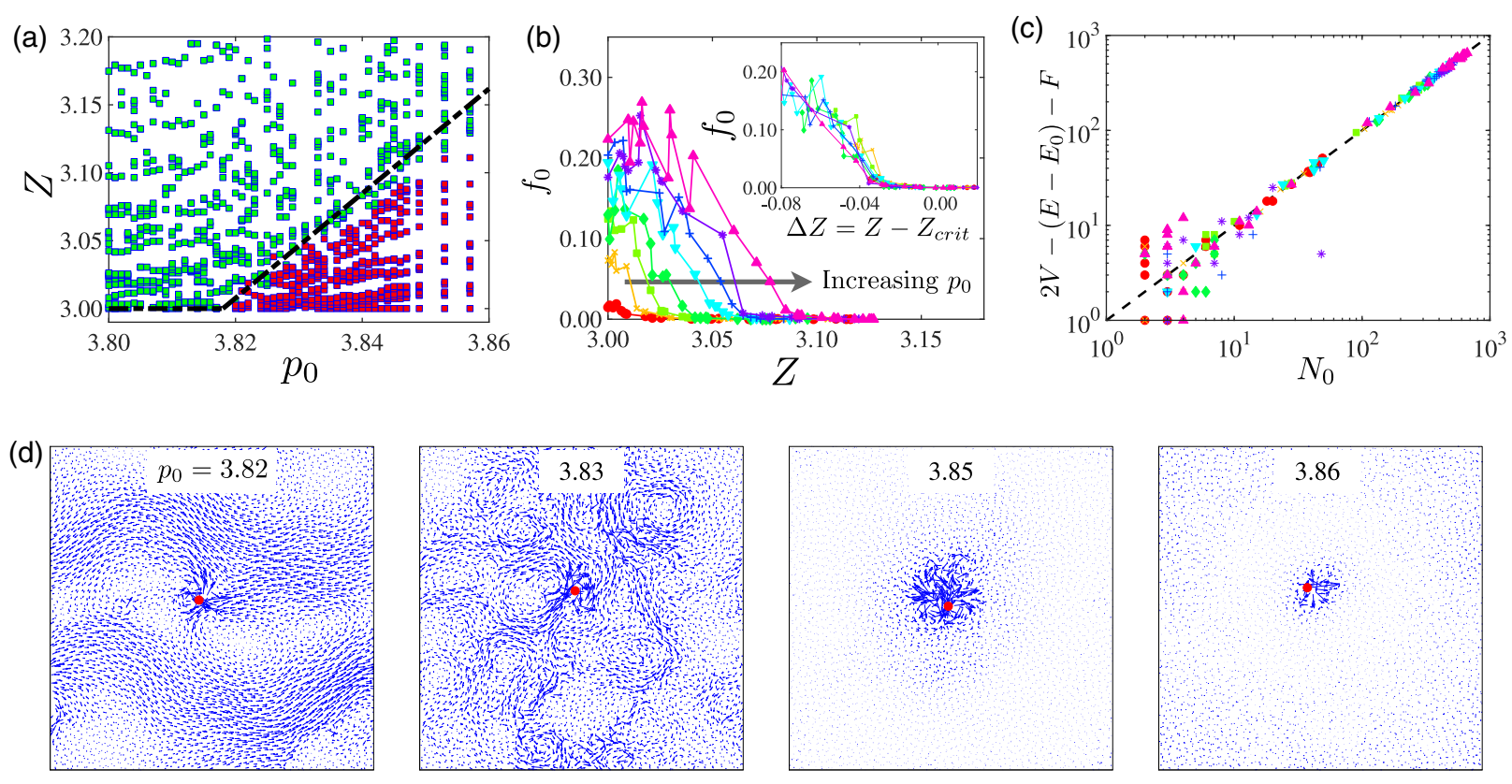

FIG. 2. Tissue mechanical properties. (a) The density of higher-order vertices $Z$ and the nondimensional cell shape index $p_{0}$ together control the mechanical rigidity of the tissue. Here, green data points indicate states with no floppy modes, i.e., mechanically rigid, and purple points highlight states with a finite number of floppy modes and zero shear modulus. When only trijunctions are allowed $(Z=3)$, rigidity occurs only for tissues with $p_{0} \leq p_{0}^{*}$. However, when a sufficient number of higher-order vertices are present $(Z>3)$, tissues can be rigidified even at $p_{0}>p_{0}^{*}$. The dashed line indicates the phase boundary between rigid and fluid states, given by $Z_{\text {crit }}\left(p_{0}\right)$ [Eq. (4)]. (b) The fraction of floppy modes $f_{0}$ as a function of $Z$ at various values of $p_{0}$ (from left to right, $p 0=3.829,3.83,3.832$, $3.834,3.836,3.838,3.84$, and 3.842). Inset: When the same data from (b) are plotted as a function of $\Delta Z=Z-Z_{\text {crit }}\left(p_{0}\right)$, all data collapse onto a single universal curve, indicating that the rigidity transition occurs at a line of critical points given by $Z_{\text {crit }}\left(p_{0}\right)$. (c) For the data points in (b), the actual number of floppy modes is compared against the prediction from the Maxwell constraint counting [Eq. (8)]. (d) Response of the tissue after a single edge collapse at $Z=3$. The displacement vector fields of all vertices are shown. The red dot indicates the location of the collapsed edge near the center of the tissue.

$N_{0}=2 V-2 F=(4-Z) V$, which deviates from the above numerical evidence and suggests that the floppy modes should always be present as long as $Z<4$. To accurately perform constraint counting [61], we apply the rank-nullity relation to the Hessian matrix:

$$
N_{0} \equiv \operatorname{nullity}(M)=\overbrace{2 V}^{\text {d.o.f. }}-\overbrace{\operatorname{rank}(M)}^{\text {no. of indep. constraints }}
$$

and count the number of independent constraints for mechanical equilibrium by calculating $\operatorname{rank}(M)$.

To illustrate how this result applies to the vertex model, we consider the Hessian of the energy without the area contribution, i.e., set $K_{A}=0$ in Eq. (1). A complete calculation for $\operatorname{rank}(M)$ for an arbitrary value of $K_{A}$ is performed in Appendix C. To calculate its rank, we first rewrite the Hessian [Eq. (3)]:

$$
M_{i \mu, j \nu}=K_{P}\left[\sum_{\alpha=1}^{F} \frac{\partial p_{\alpha}}{\partial R_{i \mu}} \frac{\partial p_{\alpha}}{\partial R_{j \nu}}+\sum_{m=1}^{E} \tau_{m} \frac{\partial^{2} l_{m}}{\partial R_{i \mu} \partial R_{j \nu}}\right] .
$$

The first term in Eq. (6) is positive definite and contributes a total count of $F$ to $\operatorname{rank}(M)$. The second term sums over all $E$ edges where $\tau_{m}=\left(p_{\alpha}-p_{0}\right)+\left(p_{\beta}-p_{0}\right)$ is the mechanical line tension [10] for an edge $m$ shared by cell $\alpha$ and $\beta$ and $l_{m}$ is the edge length. The second term in Eq. (6) can be rewritten as

$$
\sum_{m=1}^{E} \frac{\tau_{m}}{l_{m}} \hat{e}_{m}^{\perp} \hat{e}_{m}^{\perp}
$$

where $\hat{e}_{m}^{\perp}=\mathcal{R}(\pi / 2) \hat{e}_{m}$ is a $90^{\circ}$ rotation of the edge unit vector $\hat{e}_{m}=\mathbf{I}_{m} / l_{m}$. Therefore, terms in this sum [Eq. (7)] are positive definite only for edges with $\tau_{m}>0$. As a consequence, only edges with a positive tension contribute to $\operatorname{rank}(M)$, which would result in $E-E_{0}$ number of independent constraints, where $E_{0}$ is the number of edges with zero tension $\tau_{m}=0$.

Together, we obtain the rank of the Hessian matrix to be $F+E-E_{0}$ and the Maxwell rigidity criterion for the vertex model

$$
N_{0}=\overbrace{2 V}^{\text {d.o.f. }}-\overbrace{\left[\left(E-E_{0}\right)+F\right]}^{\text {no. of indep. constraints }} .
$$

Equation (8) means that both changing the topology of the network (e.g., changing $Z, E$ ) or changing the mechanical state $\left(E_{0}\right)$ can influence the rigidity of the tissue. For example, at $Z=3$, the Euler formula dictates $2 V=E+F$ 
and, hence, $N_{0}=E_{0}$, which suggests that zero modes emerge due to edges with zero tension.

Next, we put this generalized Maxwell relation to the test by directly comparing the predicted $N_{0}$ using Eq. (8) to the number of zero modes calculated from the Hessian for different $Z$ and $p_{0}$ in the fluid phase. We obtain an excellent agreement between the theoretical prediction and simulation data [Fig. 2(c)]. The agreement between the two measures in Fig. 2(c) implies that the entire rigidity line in Eq. (4) is isostatic; i.e., the number of d.o.f. matches the number of constraints. The slope of this rigidity line can be obtained through a mean-field model proposed in Appendix B, which is plotted as the dashed line in Fig. 2(a). There are several special cases of constraint counting which are detailed in Appendix C and Table I. First, there is a fixed upper limit to the number of independent constraints, i.e., that they cannot exceed the d.o.f. [i.e., $\operatorname{rank}(M) \leq 2 V$ ]. There is also a lower threshold on the number of constraints. When $K_{A}=0$, the number of constraints cannot drop below $F$, which means that, when $K_{A}=0$, it is not possible for a tissue to have zero modes when $Z=6$. When $K_{A}>0$, the number of constraints cannot drop below $2 F$, which means that a tissue will always be rigid for a state with $Z \geq 4$.

\section{B. Nonlocal response near the transition}

The rigidity transition is associated with a diverging mechanical length scale at the transition. As higher-order vertices are created using the edge collapse move, each move results in a topology change in the network where $V \rightarrow V-1, E \rightarrow E-1$, and $Z \rightarrow Z+(Z-2) /(V-1)$. These changes reduce the number of zero modes by modifying the d.o.f. and constraints according to Eq. (8). Furthermore, rosettes and $T 1$ junctions change the mean polygon geometry and induce tension, which has nonlocal effects on other cells at a distance. To capture this effect, we start from a state at $Z=3$ and measure the tissue response to an edge collapse that creates a single fourfold vertex. Figure 2(d) shows the displacement map of all vertices in response to a single edge collapse. Closer to the transition point of $p_{0}=3.81$, the response is highly nonlocal and involves a majority of vertices, with the displacement vector forming spatially extended swirl-like patterns. At $p_{0}$ values further away from the transition, the response is more localized. We quantify this growing length scale closer to $p_{0}^{*}$ by a spatial correlation function of the displacement field $C_{\mathbf{d}}(r)=\langle\mathbf{d}(0) \cdot \mathbf{d}(\mathbf{r})\rangle$ shown in Fig. 10(a). This growing length scale is highly reminiscent of the diverging dynamical length scale approaching a jamming or glass transition [62].
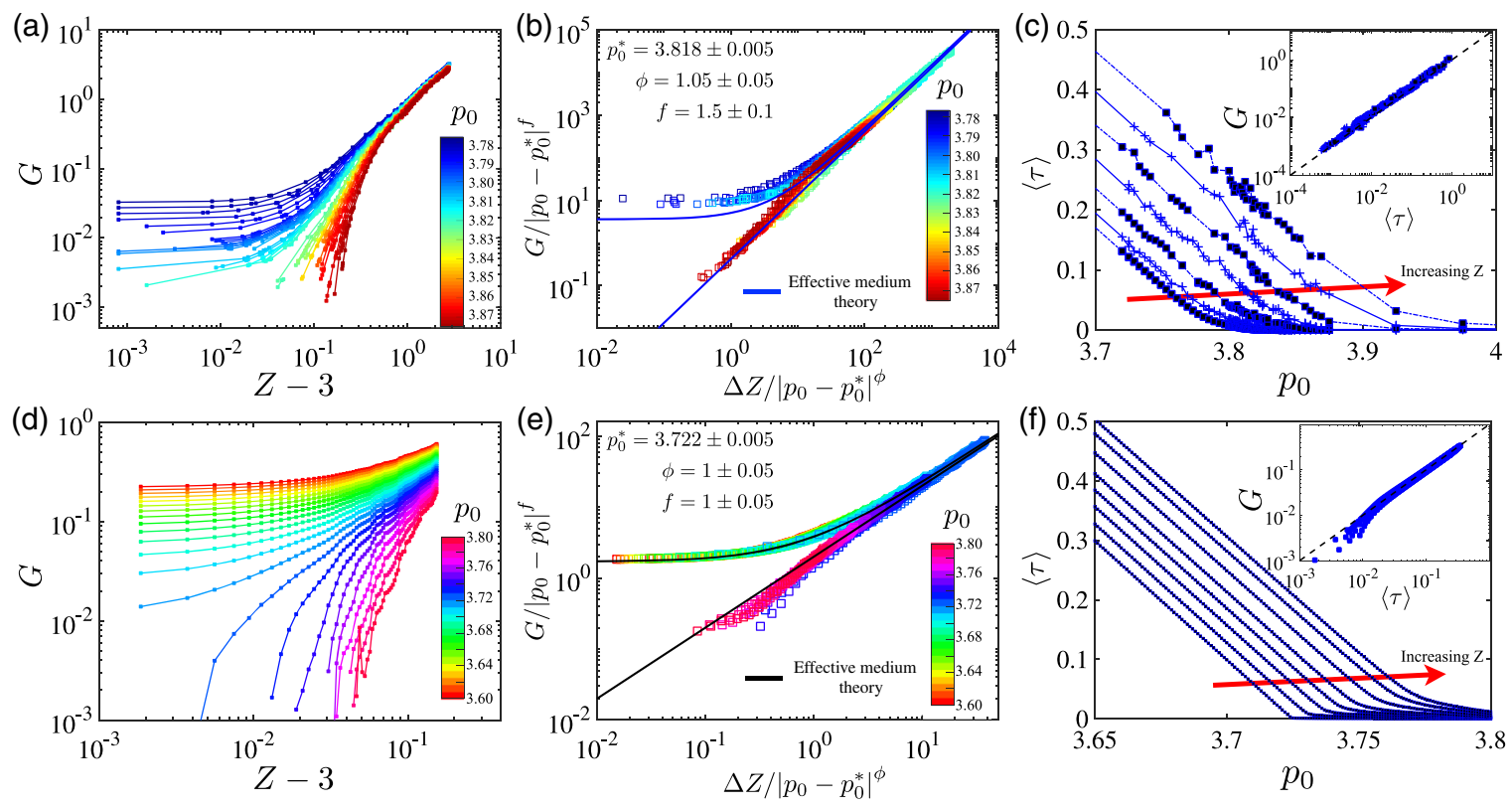

FIG. 3. Shear modulus scaling in the rigid regime. (a) The shear modulus $G$ as a function of $Z-3$ for a range of $p_{0}$ values. Here, the initial states at $Z=3$ are disordered. (b) When data from (a) are replotted according to the critical scaling ansatz [Eq. (9)], they collapse onto two distinct branches. The solid lines are predictions from the effective medium theory (EMT) [Eq. (18)]. (c) Average tension of the tissue as a function of $p_{0}$ for various values of $Z$ as indicated by the red arrow. Here, $Z=3,3.2,3.4,3.6,3.8,4,4.2$. The inset shows that the shear modulus is always a linear function of the tension, regardless of the value of $Z$. The dashed line indicates a linear relationship. (d) The shear modulus as a function of $Z-3$ for a range of $p_{0}$ values. Here, the initial states at $Z=3$ are an ordered hexagonal packing. At $Z=3$, the rigidity transition occurs at $p_{0}=p_{0} * \approx 3.722$ [2]. (e) Rescaled shear modulus data according to the scaling ansatz [Eq. (9)] show that all data collapse onto two distinct branches. The black solid lines are predictions from the effective medium theory [Eq. (18)]. (f) Average tension of the tissue as a function of $p_{0}$ for various values of $Z$ as indicated by the red arrow. Here, $Z=3,3.03$, $3.05,3.07,3.1,3.12,3.14,3.17$. The inset shows that the shear modulus is always a linear function of the tension, regardless of the value of $Z$. Here, the initial states at $Z=3$ are an ordered hexagonal packing. The dashed line indicates a linear relationship. 


\section{Critical scaling of the shear modulus}

We next probe the tissue mechanics within the rigid phase by analyzing the shear modulus. The shear modulus $G$ (defined in Appendix A) as a function of $Z$ for different values of $p_{0}$ is shown in Fig. 3(a). The functional dependence of $G$ on $Z$ separates into two regimes based on the value $p_{0}$. For $p_{0} \leq p_{0}^{*}, G$ is finite as $Z \rightarrow 3$ and increases with increasing $Z$. For $p_{0}>p_{0}^{*}$, as expected from the behavior of zero modes, $G$ vanishes at the rigidity transition line $Z_{\text {crit }}\left(p_{0}\right)$. In the limit of large $Z, G$ becomes less dependent on $Z$.

Given the hallmarks of a critical point observed for $\left(p_{0}=p_{0}^{*}, Z=3\right)[8]$ and the rapidly growing correlation length scale near the transition, we propose a criticalscaling ansatz for the shear modulus:

$$
G=\left|p_{0}-p_{0}^{*}\right|^{f} g_{ \pm}\left(\frac{\Delta Z}{\left|p_{0}-p_{0}^{*}\right|^{\phi}}\right) .
$$

$g_{+}(y)$ and $g_{-}(y)$ are the branches of the crossover scaling function for $p_{0} \leq p_{0}^{*}$ and $p_{0}>p_{0}^{*}$, respectively. Here, $y=$ $\Delta Z /\left|p_{0}-p_{0}^{*}\right|^{\phi}$ serves as the crossover scaling variable with exponent $\phi$. In Fig. 3(b), we replot all data using the rescaled variables $G /\left|p_{0}-p_{0}^{*}\right|^{f}$ and $\Delta Z /\left|p_{0}-p_{0}^{*}\right|^{\phi}$. The best collapse is obtained with exponents $f=1.5 \pm 0.1$ and $\phi=1.05 \pm 0.05$. Furthermore, the branches of the crossover scaling function lead to two distinct mechanical regimes: (I) When $p_{0} \leq p_{0}^{*}$, in the limit of $y \ll 1, g_{-}(y)=$ const or, equivalently, $G \propto\left|p_{0}-p_{0}^{*}\right|^{f}$. (II) When $y \gg 1$, the two branches merge or $g_{-}(y)=g_{+}(y) \propto y^{f / \phi}$, which means the shear modulus becomes independent of cell shapes and depends only on the network topology or $G \propto \Delta Z^{f / \phi}$.

We also perform the scaling analysis on states initialized from the hexagonal tiling. We calculate $G$ for all data shown in Fig. 9 and plot them as a function of $Z$ and $p_{0}$ values in Figs. 3(d) and 3(e). Testing the same scaling ansatz [Eq. (9)] gives a good scaling collapse and yields $f=1 \pm 0.05, \phi=1 \pm 0.05$, and $p_{0}^{*} \approx 3.722$.

\section{EFFECTIVE MEDIUM THEORY}

To better understand the scaling relations for a shear modulus, we develop an effective medium theory (EMT) [63-69] near the critical point $\left(Z=3, p_{0}^{*}\right)$. To capture the nature of the tension-induced rigidity, we map the random tension network described by the Hessian in Eq. (6) to a uniformly stressed medium whose Hessian is given by

$$
\overline{\mathcal{M}}=\mathcal{M}^{\text {topo }}+k_{\mathrm{eff}} \mathcal{M}^{\mathrm{ss}} .
$$

Here, $\mathcal{M}^{\text {topo }}$ maps to the Hessian term dependent only on the topology of the tissue network which is given by the first term in Eq. (6), and $\mathcal{M}^{\text {ss }}$ maps to the tensiondependent term [second term in Eq. (6)]. $k_{\text {eff }}$ is the effective tension, uniform on all edges. Now, replacing the tension on edge $m$ with $k_{m}=\tau_{m} / l_{m}$, a random variable obeying the probability distribution

$$
p(k)=P \delta(k-\bar{k})+(1-P) \delta(k),
$$

which characterizes both edges of zero internal tension and the ones of the order of $\bar{k}$ (a more general distribution is considered in Appendix D), results in a scattering potential:

$\mathcal{V}=\mathcal{M}_{m}-\overline{\mathcal{M}}= \begin{cases}\left(k_{m}-k_{\mathrm{eff}}\right) e_{m \mu}^{\perp} e_{m \nu}^{\perp} & \text { if } i=j \in \partial m, \\ -\left(k_{m}-k_{\mathrm{eff}}\right) e_{m \mu}^{\perp} e_{m \nu}^{\perp} & \text { if } i \neq j \in \partial m, \\ 0 & \text { otherwise }\end{cases}$

where $\partial m$ is the set of vertices defining the edge $m$.

The Green's function of the perturbed system $\mathcal{G}$ can be written in terms of the Green's function of the effective medium $\overline{\mathcal{G}}=\left(\overline{\mathcal{M}}-\omega^{2} \mathcal{I}\right)^{-1}$ :

$$
\mathcal{G}=\left(\mathcal{M}-\omega^{2} \mathcal{I}\right)^{-1}=\overline{\mathcal{G}}+\overline{\mathcal{G}} \mathcal{T} \overline{\mathcal{G}},
$$

where $\mathcal{T}=-\mathcal{V} \sum_{n \geq 0}(-\overline{\mathcal{G}} \mathcal{V})^{n}$ sums over all multiplescattering contributions of the edge $m$. The EMT assumes the effective medium resembles the random medium if on average the replacement does not effect the mechanical propagation, i.e., $\int p(k) \mathcal{G}(k) d k=\overline{\mathcal{G}}$ :

$$
P \mathcal{T}\left(k_{m}=\bar{k}\right)+(1-P) \mathcal{T}\left(k_{m}=0\right)=0 .
$$

This assumption results in the self-consistency equation given by

$$
P \frac{\bar{k}-k_{\mathrm{eff}}}{1+\left(\bar{k}-k_{\mathrm{eff}}\right) G_{m}}+(1-P) \frac{-k_{\mathrm{eff}}}{1-k_{\mathrm{eff}} G_{m}}=0,
$$

where $G_{m}=\langle\overline{\mathcal{G}}\rangle_{m}=2 \hat{e}_{m}^{\perp} \cdot \overline{\mathcal{G}}_{i i} \cdot \hat{e}_{m}^{\perp}-2 \hat{e}_{m}^{\perp} \cdot \overline{\mathcal{G}}_{i j} \cdot \hat{e}_{m}^{\perp}$. The perimeters contribute $F / V=[(Z-2) / 2]$ independent constraints on each vertex, so, on vertex $i, \operatorname{tr}\langle\overline{\mathcal{G}} \overline{\mathcal{M}}\rangle_{i, \omega=0}=$ $[(Z-2) / 2]+k_{\mathrm{eff}}(Z / 2) G_{m}=d$. So $G_{m}=\left(h / k_{\mathrm{eff}}\right)$, where $h=[(6-Z) / Z]$ (a more strict derivation is done for the honeycomb lattice in Appendix D). Inserting it into the selfconsistency equation (15), we have the effective tension

$$
k_{\text {eff }}= \begin{cases}\bar{k} \frac{P-h}{1-h} & P \geq P_{c}=h=\frac{6-Z}{Z}, \\ 0 & \text { otherwise }\end{cases}
$$

As $P \in[0,1], Z^{*}=3$ is the minimal coordination for a vertex network to be stabilized by tension. The number of zero modes is $N_{0}=E\left(P_{c}-P\right)=E[(6-Z) / Z]-E P=$ $2 V-F-\left(E-E_{0}\right)$.

The elastic modulus is related to the Fourier transform of the Green's function as $\lim _{\mathbf{q} \rightarrow 0} C_{i j k l} q_{i} q_{k} \mathcal{G}_{j l, \omega=0}=1$ [70]. 
As shown above, near the rigidity threshold, the Green's function of the effective medium is singular as $\overline{\mathcal{G}} \sim k_{\text {eff }}^{-1} q^{-2}$. So the shear modulus scales as the effective tension, $G \propto k_{\text {eff }} \sim \bar{k}$.

\section{A. Effective medium theory predicts the critical scaling observed in simulations}

As shown in the insets in Figs. 3(c) and 3(f), the theory predicts that the shear modulus scales as the effective tension on edges, $G \sim k_{\text {eff }}$, which is determined by a selfconsistency equation and proportional to the mean tension in the network $\bar{k}$ when the Maxwell criterion is saturated $N_{0}=0$. In the limit $\bar{k} \rightarrow 0$, the average tension vanishes with the geometric frustration,

$$
G \propto \bar{k} \sim\left|p_{0}-p_{0}^{*}(Z)\right|^{f} .
$$

Inserting Eq. (4), the scaling relation for the modulus

$G \propto \begin{cases}\left|p_{0}-p_{0}^{*}\right|^{f}\left[1+\frac{Z-3}{\mathcal{B}\left|p_{0}-p_{0}^{*}\right|}\right]^{f} & p_{0}<p_{0}^{*}, \\ \left|p_{0}-p_{0}^{*}\right|^{f}\left[\frac{Z-Z_{\text {crit }}\left(p_{0}\right)}{\mathcal{B}\left|p_{0}-p_{0}^{*}\right|}\right]^{f} & p_{0}>p_{0}^{*} .\end{cases}$

This result implies that $\phi=1$. The EMT also gives the exponent $f$. On one hand, from the definition of the energy Eq. (1), we have $U / F \sim \bar{k}^{2}$ when $K_{A}=0$. On the other, the energy $U$ is a smooth function of $p_{0}$ vanishing at threshold $p_{0}^{*}$, so that we can Taylor expand it near $p_{0}^{*}: U / F=$ $c_{2}\left(p_{0}^{*}-p_{0}\right)^{2}+c_{3}\left(p_{0}^{*}-p_{0}\right)^{3}+\cdots$. When the vertex network does not relax, for example, on the ordered hexagonal cells, the actual perimeter $p_{\alpha}$ stays as $p_{0}^{*}$ as $p_{0}$ decreases, so $\bar{k} \propto p_{0}^{*}-p_{0}, f=1$. When the network does relax, the energy vanishes at the quadratic order, $c_{2}=0$, due to the floppy nature of the unstressed network. To the lowest order, $U / F \propto\left[p_{0}^{*}(Z)-p_{0}\right]^{3}$, which implies an exponent of $f=$ $3 / 2$. This result explains the different exponents found in the disordered and ordered cases, as also confirmed numerically in Figs. 3(c) and 3(f). The EMT prediction Eq. (18) with the only fitting parameter $p_{0}^{*}$, shown as solid lines in Figs. 3(b) and 3(e), agrees well with the numerical results.

\section{DISCUSSION AND CONCLUSIONS}

In this work, we have revealed that the topology of the tissue network (controlled by the average vertex coordination $Z$ ) and the intracellular tensions (controlled by the parameter $p_{0}$ ) can greatly influence the rigidity of the tissue. The interplay of these parameters gives rise to a fluid-to-solid transition as well as different mechanical regimes in the solid phase. The rich set of behaviors is summarized in a phase diagram (Fig. 4). Until now, there has been a lack of a theoretical explanation for the recently observed jamming transitions and rigidity in dense tissues [8]. Whereas conventional wisdom on constraint counting

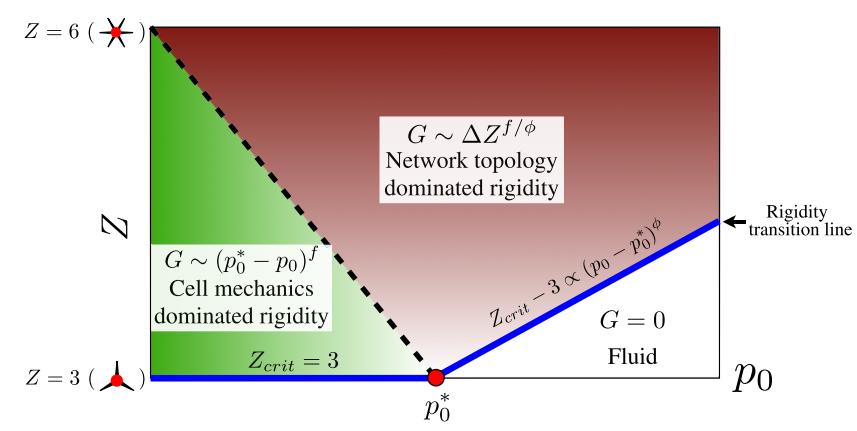

FIG. 4. A phase diagram in $Z-p_{0}$ space. The critical scaling of $G$ suggests three distinct mechanical regimes. When $\Delta Z \ll$ $\left|p_{0}-p_{0}^{*}\right|^{\phi}$, the tissue is rigid with a shear modulus that is dominated by cellular mechanics which is encoded in the shape index $p_{0} . \Delta Z \gg\left|p_{0}-p_{0}^{*}\right|^{\phi}$, the topology of the cell network dominates tissue rigidity where the shear modulus has a powerlaw dependence on the average vertex coordination $Z$. The tissue becomes fluidlike as floppy modes emerge at the line given by $Z_{\text {crit }}\left(p_{0}\right)$.

would erroneously suggest that a tissue should always be fluidlike, this work explains why $Z=3$ states can be stabilized. It further offers a unifying perspective on why a rigidity transition can be expected at all in a cellular material by using an generalized Maxwell constraint counting approach.

This work makes several experimentally verifiable quantitative predictions for cell shape and tissue mechanics. First, our model provides a criterion to determine the rigidity of a tissue from direct measures of cellular geometry. As the values of $Z$, cell perimeters $\{P\}$, and cell areas $\{A\}$ are experimentally accessible, Eq. (B1) predicts that a tissue should be fluidlike if $p \equiv\langle P / \sqrt{A}\rangle\rangle$ $p_{0}(Z=3)+\mathcal{B}^{-1}(Z-3)$ and rigid if $p \equiv\langle P / \sqrt{A}\rangle \leq$ $p_{0}(Z=3)+\mathcal{B}^{-1}(Z-3)$, where $p_{0}(Z=3) \approx 3.8$ and $\mathcal{B} \approx 3.85$ follow from the theoretical results of this paper. Second, the scaling relation of how the tension in the tissue would grow with the creation of higher-order vertices, $\langle\tau\rangle \propto(Z-3)^{1.5}$, can be verified with tension measured in laser-ablation experiments $[31,34,71]$ or mechanical inference methods [36,72-76].

Our result provides a direct understanding of the observed fluidization of epithelium in Drosophila embryo development [57]. Preliminary work [77] on measuring the shape index (ratio between cell perimeter and $\sqrt{\text { area }}$ ) as well as the coordination number $Z$ in the developing Drosophila embryo suggests that the tissue is on the solid side but remains close to the solid-liquid phase boundary before gastrulation; however, it crosses the boundary and transitions into a fluidlike state when a ventral furrow forms and dives far into the liquid phase during the fast germ-band extension associated with rosette forming. Furthermore, our predictions that rigidity is lost and shear modulus vanishing in the fluid phase is consistent with elastic moduli measured in Ref. [57]. These observations [57,77] suggest that rigidity 
vanishes at the formation of a ventral furrow and the onset of a fast germ-band extension event.

We also predict a high degree of correlation between higher-order vertices and adjacent edge tensions. A representative example is shown in Figs. 5(a) and 5(b). Here, $T 1$ junctions and rosettes are marked in blue, and cell edges are drawn with widths proportional to the edge tensions. We consistently observe higher-order vertices coincide with nearby high edge tensions. To quantify this correlation, edges are grouped according to the degree of their adjacent vertices $\left(Z_{1}\right.$ and $\left.Z_{2}\right)$. This grouping results in three different categories of edges: (i) edges associated with only trijunctions $\left(Z_{1}=Z_{2}=3\right.$ ), (ii) edges associated with $T 1$ junctions (at least one adjacent vertex is a $T 1$ junction), and (iii) edges associated with rosettes (at least one adjacent vertex is a rosette; i.e., $Z_{1}>4$ or $Z_{2}>4$ ). The relative fractions for these edge types are plotted as functions of $Z$ in the inset in Fig. 5(c). Interestingly, the statistics show very good agreement (Fig. 12) with the cell topologies measured during Drosophila embryo elongation [33]. Next, for edge types, we compare their tensions to the mean tension of the entire tissue, which is shown in Fig. 5(c). This comparison predicts that, as $Z$ increases, the edges near higher-order vertices consistently carry more tension compared to trijunction vertices. In particular, at the onset of higher-order vertices appearing, tensions associated with $T 1$ junctions are in the range of $1.5 \pm 0.3$ times the tension near trijunctions. Again, these numbers match up well with the experimental results from Drosophila embryo elongation, where tensions associated with edges pointing along the anterior-posterior (AP) direction are typically 1.7 times [34] compared to edges pointing in the dorsal-ventral (DV) direction. While a more systematic study is warranted to model the elongation processes in Drosophila development, our minimal model

(a)

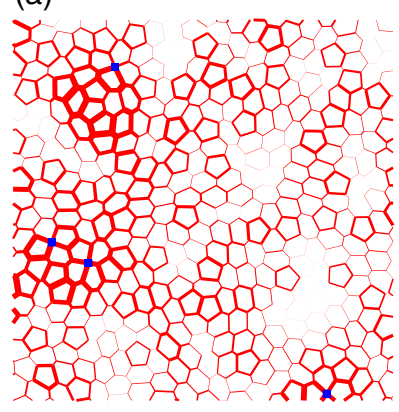

(b)

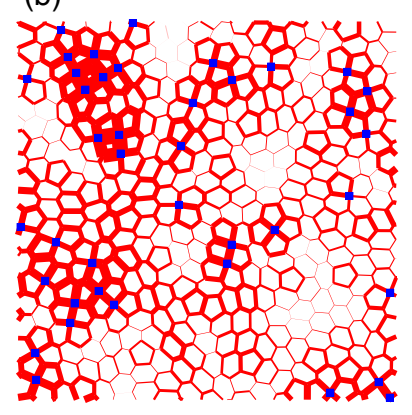

suggests that the correlations between junctional tension and cellular topologies may be more universal. For future work, we will build on these general results to specifically model the feedback mechanism that leads to rosette formation in Drosophila embryogenesis, where both an increased rosette count and increased tensions are predicted to emerge from the myosin positive-feedback loop [34,78]. Finally, the criticality of this rigidity transition results in a nonlocal response to perturbations near the critical point, as shown in Fig. 2(d). We thus expect the laser ablation [78] of cell edges to induce a similar long-range effect to collapsing edges near the onset of tissue rigidity. The spatial extension of the response could even be used as an indicator of how far the tissue is from the rigidity transition.

In this work, we have focused mainly on the mechanics of tissues with stable rosettes. However, rosettes in actual tissues can vary greatly in their lifetime. This variation ranges from rosettes that are transient and resolve quickly to form new structures to rosettes that persist for an extended period of time or may not resolve at all. For example, the lifetime of higher-order vertices is thought to be linked to the Hippo pathway and the force-sensitive protein Ajuba $[59,79,80]$. Our predictions are therefore applicable to the mechanical response of the tissue at timescales shorter than the rosette resolution time. In the version of the vertex model here, we have not included biological feedback of cells in response to mechanical stress. However, in Drosophila embryo elongation, for example, myosin motors can slide more on actin in response to higher tensions, and, as a result, the observed cell perimeters can change [12]. At the same time, myosin recruitment is stimulated to sustain the perimeter at high tensions. These feedback events occur at a characteristic timescale corresponding to the relaxation and resolution of rosettes. We would expect a breakdown of

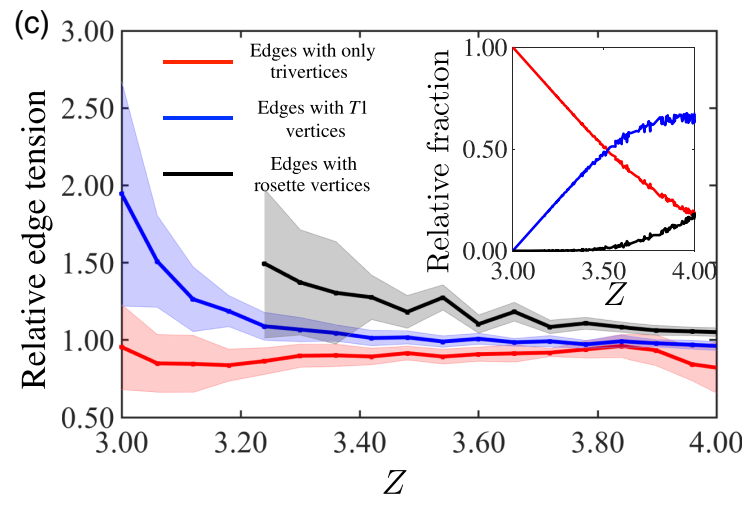

FIG. 5. Spatial correlation between higher-order vertices and tensions. (a) At $p_{0}=3.8$ and $Z \approx 3.01$, a few higher-order vertices are present, and their creation causes the tension in the tissue to increase from $Z=3$. Here, higher-order vertices are indicated by blue markers, and the thickness of cell edges is proportional to the tension magnitude. (b) Holding $p_{0}=3.8$ constant and further introducing higher-order vertices brings the tissue to $Z \approx 3.06$. There is a significant degree of correlation between higher-order vertices and heightened local tensions (c) Relative edge tension due trijunctions (red), $T 1$ junctions (blue), and rosettes (black) plotted as a function of $Z$ at fixed $p_{0}=3.8$. The solid lines show the median values of each group, and shaded regions indicate the standard deviation. The relative tension is defined as the edge tension scaled by the mean tension in the entire tissue $\langle\tau(Z)\rangle$, which scales as $(Z-3)^{1.5}$. Inset: The relative populations of tri-, $T 1$, and rosette vertices as a function of $Z$. 
the static rigidity theory in these regimes. For the Drosophila embryo, this corresponds to a timescale of several minutes [81]. Modeling dynamical movements and transient formation + resolution of rosettes will be an interesting avenue for future research.

Another important aspect of this work is the use of a generalized Maxwell constraint counting argument to explain why rigidity transitions can occur in tissues in general. Whereas conventional counting arguments suggest [82] that the vertex model is always underconstrained, we show such not to be the case through an accurate counting of mechanical constraints. For example, conventional constraint counting would predict that a cellular material should be stable only for $Z>4$. However, most epithelial tissues can be stable at precisely $Z=3$. This work explains why $Z=3$ states are stabilized and further offers a unifying perspective on why a rigidity transition can be expected at all in a cellular material by using a Maxwell constraint counting approach. Our understanding of this rigidity transition is based on a generalized Maxwell counting that properly counts the critical role of tension. It is a natural extension of the stress-driven rigidity transition in packings and fiber networks close to the critical topology with $Z=2 d[66,83-$ 85]. The appearance of the tension can also be viewed as a result of the geometric incompatibility of the material metric as studied in a continuum treatment of the vertex model by Moshe and co-workers [18]. The similar insight of tension and geometric compatibility was also proposed in a recent study by Merkel and Manning [11] on the 3D Voronoi-based cell model, a variation of the vertex model $[9,17,86]$.

The constraint counting developed here can be easily generalized to the Voronoi-based cell models. In those models, the d.o.f. are cell centers (whose number is $2 F$ ) rather than cell vertices, and the cell shapes are obtained from a Voronoi tessellation of the cell centers. For the same energy in Eq. (1), the number of constraints is the same as that given by Eq. (8), i.e., $\max \left(2 F, E-E_{0}+F\right)$, which leads to a constraint counting that is given by $N_{0}-N_{s s}=2 F-\max \left(2 F, E-E_{0}+F\right)$. When edges carry tensions and the number of constraints is greater than the d.o.f. $E-E_{0}+F>2 F$, we expect there to be redundant constraints which will result in $N_{s s}$ states of selfstress. Interestingly, when $2 F>E-E_{0}+F$, the counting leads to $N_{0}=0$, indicating that the system is always marginal and, thus, the lack of a transition to fluid in the linear response, as recently observed and studied in Ref. [87]. However, the solid-fluid transition is still evidently shown in the Voronoi-based model with selfpropelling cells $[9,24]$. The nature of this observed transition is determined by two aspects. First, there is still a transition to a self-stressed state when the number of edges under tension, $E-E_{0}$, becomes larger than the number of cells $F$ at the same threshold $p_{0}=3.81$. Second, the marginal nature of the system when $p_{0}>3.81$ makes the linear response fragile and plastic nonlinear processes
(T1 rearrangement, rosette formation, etc.) take over the response, so that the system behaves as a liquid whenever a finite energy is injected to it. This prediction is supported by ongoing work [77], which appears to show that, in the marginal range of Voronoi-based cell models, $T 1$ rearrangements can be easily triggered with a perturbation whose magnitude vanishes with the system size. This behavior could very well suggest an example where the Maxwell constraint counting and linear-response theory fail to predict the loss of the rigidity.

\section{ACKNOWLEDGMENTS}

The authors acknowledge Sebastian Streichan for providing helpful comments, suggestions, and preliminary experimental data. The authors also thank Boris Shraiman, Matthieu Wyart, Cristina Marchetti, Mark Bowick, Jen Schwarz, Jin-Ah Park, Lisa Manning, Xiaoming Mao, Frederick MacKintosh, Eric DeGiuli, Karen Kasza, and Bulbul Chakraborty for helpful discussions. This research was supported in part by National Science Foundation (NSF) Grant No. PHY-1748958, National Institutes of Health (NIH) Grant No. R25GM067110, and the Gordon and Betty Moore Foundation Grant No. 2919.01. The authors acknowledge the support of the Northeastern University Discovery Cluster.

\section{APPENDIX A: SIMULATION METHODS}

We simulate tissues composed of $N=625$ cells under periodic boundary conditions with box size $L=\sqrt{N}=25$, which is commensurate with the value of the preferred cell area of unity. We use $K_{p}=1$ for the data shown in this paper. The value of $p_{0}$ is varied between 3.6 and 4.3. We carry out the protocol using two different types of initial states at $Z=3$ : (i) random tissue networks, where cell shapes are obtained from the random Voronoi tessellations in the Voronoi-based cell model [9], and (ii) an ordered hexagonal tiling [2,4]. After initialization, the vertex positions in the tissue are evolved to the energy minimum using the Broyden-Fletcher-Goldfarb-Shanno method [88] until the residual force magnitude on all vertices is less than $10^{-8}$. The simulations methods are based on Ref. [8].

The shear modulus is calculated by considering the linear response of the tissue to an infinitesimal affine strain $\gamma$. It is given by [89]

$G=G_{\text {affine }}-G_{\text {nonaffine }}=\left.\frac{\partial^{2} U}{\partial \gamma^{2}}\right|_{\gamma=0}-\Xi_{i \mu} M_{i \mu j \nu}^{-1} \Xi_{j \nu}$.

Here, $\Xi_{i \mu}$ is the derivative of the force on vertex $i$ with respect to the strain, given by

$$
\left.\Xi_{i \mu} \equiv \frac{\partial^{2} U}{\partial \gamma \partial r_{i \mu}}\right|_{\gamma=0} .
$$




\section{APPENDIX B: MEAN-FIELD MODEL FOR DETERMINING THE SLOPE OF THE RIGIDITY LINE}

The rigidity transition line in the $\left(p_{0}, Z\right)$ plane can be expressed as Eq. (4) or its inverse

$$
p_{0}^{*}(Z)=p_{0}^{*}(Z=3)+\mathcal{B}^{-1}(Z-3),
$$

where $\mathcal{B}^{-1}=\partial p_{0}^{*}(Z) /\left.\partial Z\right|_{Z=3}$, which captures how the critical preferred perimeter changes when the number of edges per cell changes. We then take a mean-field approach and replace $p_{0}^{*}(Z)$ with the perimeter of a regular $n$-sided polygon (with area of unity) of $n=2 Z /(Z-2)$ sides:

$$
P_{n}=2 \sqrt{n} \tan (\pi / n)
$$

to obtain $\mathcal{B}^{-1}=\partial P_{n} /\left.\partial Z\right|_{Z=3} \approx 0.260$. This mean-field approximation serves well to predict the slope of the transition line [Fig. 2(a)]. However, the properties of regular polygons are not helpful for predicting the value of the transition point itself $\left[p_{0}^{*}(Z=3)=3.81\right.$ (disordered), 3.72(hex)], which is an emergent collective property of the system.

\section{APPENDIX C: GENERALIZED MAXWELL CONSTRAINT COUNTING FOR THE VERTEX MODEL}

\section{Hessian (dynamical matrix) of the vertex model}

We begin by calculating the Hessian matrix of the total energy, which is given by Eq. (1). Now, since $\sum_{\alpha} A_{\alpha}=$ const for a confluent tissue not undergoing cell number changes, it is equivalent to work with the simpler form of energy:

$$
U=\frac{1}{2} \sum_{\alpha=1}^{F}\left[K_{A} A_{\alpha}^{2}+K_{P}\left(P_{\alpha}-P_{0}\right)^{2}\right] .
$$

In Eq. (C1), we also multiply the energy by a factor of $1 / 2$ for convenience when taking derivatives.

The network of cells is given by vertices (at positions $\left\{\mathbf{R}_{i}\right\}$ ) and edges (specified by edge vectors $\mathbf{L}_{m}$ ). The relation between edges and vertices is given by the directed adjacency matrix $g_{m i}$. Edge vectors can be calculated using

$$
\mathbf{L}_{m}=\sum_{i} g_{m i} \mathbf{R}_{i}
$$

where $g_{m i}$ is nonzero if vertex $i$ is on edge $m$; it is +1 if vertex $i$ forms the head of edge vector $m$ and -1 if it is the tail. The relation between edges and facets (cells) is given by edge-facet adjacency matrices $h_{m \alpha}$, which is $+1(-1)$ if edge $m$ goes counterclockwise (clockwise) around facet $\alpha$ and zero otherwise. In this notation, both the area and the perimeter can be written in vertices and edges:

$$
\begin{aligned}
A_{\alpha} & =\frac{1}{4} \sum_{m, i}\left|g_{m i}\right| h_{m \alpha}\left(\mathbf{R}_{i} \times \mathbf{L}_{m}\right) \cdot \mathbf{z} \\
& =-\frac{1}{4} \sum_{m, i}\left|g_{m i}\right| h_{m \alpha} \mathbf{R}_{i} \cdot \mathbf{L}_{m}^{\perp}, \\
P_{\alpha} & =\sum_{m}\left|h_{m \alpha}\right| L_{m} .
\end{aligned}
$$

First, it is instructive to calculate the total force on each vertex, which is given by the gradient of Eq. (C1):

$f_{i \mu} \equiv-\frac{\partial U}{\partial R_{i \mu}}=\sum_{\alpha=1}^{F}\left[K_{A} A_{\alpha} \frac{\partial A_{\alpha}}{\partial R_{i \mu}}+K_{P}\left(P_{\alpha}-P_{0}\right) \frac{\partial P_{\alpha}}{\partial R_{i \mu}}\right]$.

Based on the definitions of the area and perimeter [Eq. (C3)], we obtain the geometric derivatives

$$
\begin{aligned}
\frac{\partial A_{\alpha}}{\partial R_{i \mu}} & =-\frac{1}{2} \sum_{m}\left|g_{m i}\right| h_{m \alpha} L_{m} e_{m \mu}^{\perp} \quad \text { and } \\
\frac{\partial P_{\alpha}}{\partial R_{i \mu}} & =\sum_{m}\left|h_{m \alpha}\right| g_{m i} e_{m \mu} .
\end{aligned}
$$

Therefore, using Eq. (C5), it is possible to rewrite Eq. (C4) in terms of a sum over all edges:

$f_{i \mu}=-\sum_{m=1}^{E}\left(-\frac{1}{2} K_{A} \sigma_{m} L_{m}\left|g_{m i}\right| e_{m \mu}^{\perp}+K_{P} \tau_{m} g_{m i} e_{m \mu}\right)$,

where we have separated the force on each vertex into two mutually orthogonal components: a component along the edge vector $\hat{\mathbf{e}}_{m} \equiv \mathbf{L}_{\mathbf{m}} / L_{m}$ and a component perpendicular to the edge vector given by $\hat{\mathbf{e}}_{m}^{\perp}=\mathcal{R}(\pi / 2) \hat{\mathbf{e}}_{m}$, or the edge vector rotated by $\pi / 2$. The magnitudes of force are given by

$$
\begin{aligned}
& \tau_{m}=\sum_{\alpha}\left|h_{m \alpha}\right|\left(P_{\alpha}-P_{0}\right)=\left[\left(P_{\alpha}-P_{0}\right)+\left(P_{\beta}-P_{0}\right)\right], \\
& \sigma_{m}=\sum_{\alpha} h_{m \alpha} A_{\alpha}=A_{\alpha}-A_{\beta} .
\end{aligned}
$$

$K_{P} \tau_{m}$ is the line tension due to the mismatch between the actual perimeters of cell $\alpha$ and $\beta$ from the preferred perimeter. $K_{A} \sigma_{m} / 2$ is the pressure due to the difference between the areas of cells $\alpha$ and $\beta$. 
The Hessian is a $2 V \times 2 V$ matrix given by

$$
\begin{aligned}
M_{j \nu, i \mu} \equiv & \frac{\partial U^{2}}{\partial R_{j \nu} \partial R_{i \mu}} \\
= & \sum_{m}\left[K_{P}\left(\frac{\partial \tau_{m}}{\partial R_{j \nu}} g_{m i} e_{m \mu}+\frac{\partial e_{m \mu}}{\partial R_{j \nu}} \tau_{m} g_{m i}\right)\right. \\
& \left.-\frac{1}{2} K_{A}\left(\frac{\partial \sigma_{m}}{\partial R_{j \nu}} L_{m \mu}^{\perp}\left|g_{m i}\right|+\frac{\partial L_{m \mu}^{\perp}}{\partial R_{j \nu}} \sigma_{m}\left|g_{m i}\right|\right)\right],
\end{aligned}
$$

which can be written explicitly as

$$
\begin{aligned}
M_{j \nu, i \mu}= & \sum_{m=1}^{E} \sum_{n=1}^{E}\left[K_{P} e_{n \nu} g_{n j} * H_{n m} * e_{m \mu} g_{m i}\right. \\
& +K_{P} e_{n \nu}^{\perp} g_{n j} * T_{n m} * e_{m \mu}^{\perp} g_{m i} \\
& +\frac{K_{A}}{4} e_{n \nu}^{\perp} g_{n j} * \tilde{H}_{n m} * e_{m \mu}^{\perp} g_{m i} \\
& +\frac{K_{A}}{2} e_{n \nu} g_{n j} * \Sigma_{n m} * e_{m \mu}^{\perp} g_{m i} \\
& \left.-\frac{K_{A}}{2} e_{n \nu}^{\perp} g_{n j} * \Sigma_{n m} * e_{m \mu} g_{m i}\right] .
\end{aligned}
$$

Equation (C9) has been written in a symmetric form where each term is a $(2 V \times 2 V)$ matrix decomposed into a product of three matrices $(2 V \times E)(E \times E)(E \times 2 V)$. Here, the $E \times E$ matrices are defined as

$$
\begin{aligned}
H_{n m} & =\sum_{\alpha=1}^{F}\left|h_{n \alpha} h_{m \alpha}\right|, \\
\tilde{H}_{n m} & =L_{n} L_{m} \sum_{\alpha=1}^{F} h_{n \alpha} h_{m \alpha}, \\
T_{n m} & =\frac{\tau_{m}}{L_{m}} \delta_{n m}, \\
\Sigma_{n m} & =\sigma_{m} \delta_{n m} .
\end{aligned}
$$

The matrices $H$ and $\tilde{H}$ give the relationship between edges and facets. $T$ and $\Sigma$ are diagonal matrices which give the tension and pressure, respectively, on each edge. The geometric property of edges and the relationship between edges and vertices are captured by the $2 V \times E$ matrices

$$
\begin{aligned}
& S_{n, j, \nu}^{\|}=e_{n \nu} g_{n j}, \\
& S_{n, j, \nu}^{\perp}=e_{n \nu}^{\perp} g_{n j},
\end{aligned}
$$

which are mutually independent by definition. In matrix form, Eq. (C9) can be written using Eqs. (C11) and (C10) in matrix form as a product of block matrices:

$$
\begin{aligned}
M & =S C S^{T} \\
& =\left(S^{\|} \mid S^{\perp}\right)\left(\begin{array}{c|c}
K_{P} H & -\frac{1}{2} K_{A} \Sigma \\
\hline \frac{1}{2} K_{A} \Sigma & K_{P} T+\frac{1}{4} K_{A} \tilde{H}
\end{array}\right)\left(S^{\|} \mid S^{\perp}\right)^{T} .
\end{aligned}
$$

Here, we define the augmented matrix $S \equiv\left(S^{\|} \mid S^{\perp}\right)$ and a $2 E \times 2 E$ block matrix

$$
C=\left(\begin{array}{c|c}
K_{P} H & -\frac{1}{2} K_{A} \Sigma \\
\hline \frac{1}{2} K_{A} \Sigma & K_{P} T+\frac{1}{4} K_{A} \tilde{H}
\end{array}\right) .
$$

$C$ is block antisymmetric.

\section{Constraint counting}

We apply the rank-nullity theorem to the Hessian matrix in order to accurately count the number of constraints [61]:

$$
\operatorname{rank}(M)+\operatorname{nullity}(M)=2 V .
$$

The rank of $M$ give the number of independent constraints for force balance $[61,67]$. The nullity of $M$ is the dimensionality of the null space of $M$, i.e., the number of solutions to $M\left|\delta \mathbf{R}_{i}\right\rangle=0$. These correspond to the number of ways to infinitesimally perturb the vertices such that the energy of the system does not change. This is exactly the number of zero modes in the Hessian, which we term $N_{0}$. Therefore, the constraint counting becomes

$$
\operatorname{nullity}(M)=N_{0}=\overbrace{2 V}^{\text {d.o.f. }}-\overbrace{\operatorname{rank}(M)}^{\text {no. of indep. constraints }} .
$$

To calculate the rank of $M$, we first note that it has a trivial upper bound of $2 \mathrm{~V}$ (in which case $N_{0}=0$ ). Additionally, since $M$ is a product of $S$ and $C$ and $S$ is full rank, it follows that [90] $\operatorname{rank}(M)=\operatorname{rank}(C)$, and by application of Guttman rank additivity of a block matrix [91] we can write down a general relation

$$
\begin{aligned}
\operatorname{rank}(M)= & \operatorname{rank}\left(K_{P} H\right)+\operatorname{rank}\left(K_{P} T+\frac{1}{4} K_{A} \tilde{H}\right. \\
& \left.+\frac{1}{4} K_{A}^{2} K_{P}^{-1} \Sigma H^{\dagger} \Sigma\right) .
\end{aligned}
$$

In Eq. (C16), $H^{\dagger}$ is the Morse-Penrose pseudoinverse of $H$, since below we show that it is always less than full rank and therefore not invertible. Since $H$ can be expressed as a product of matrices with lesser rank $F$ [Eq. (C10)], it always holds that $\operatorname{rank}(H)=F$. The reason that the pseudoinverse can be done without changing the actual dimension of the $C$ matrix is that one can show that the rank of $\Sigma$ is also $F$, and these $F$ directions are not independent of the $F$ directions in $H$.

In the case of $K_{A}=0, \operatorname{rank}(M)=\operatorname{rank}(H)+\operatorname{rank}(T)$. The rank of $T$ is precisely given by the number of edges 


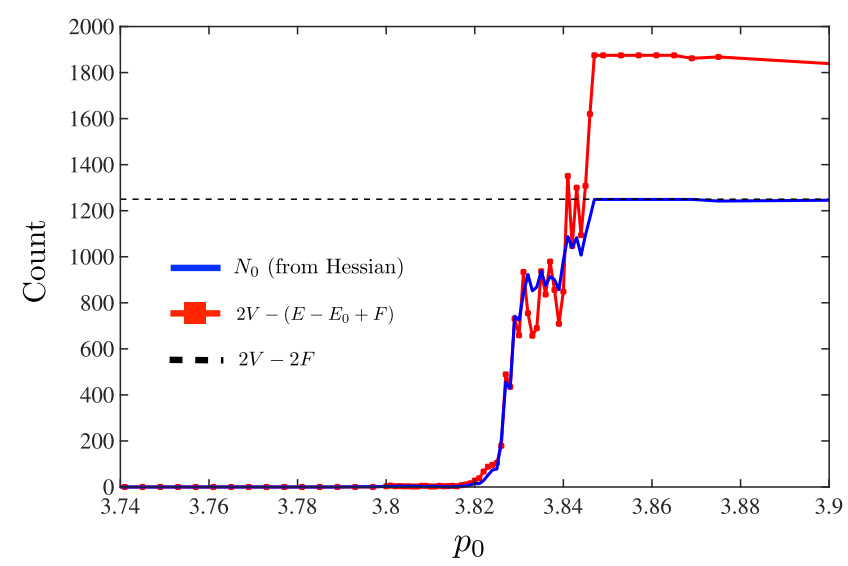

FIG. 6. Maxwell counting in the vertex model is given by $N_{0}=2 V-\max \left(2 F, E-E_{0}+F\right)$.

with $\tau_{m}>0$ [Eq. (C10)], which we define as $E-E_{0}$ in the main text. Hence, the constraint counting for when $K_{A}=0$ is given by

$$
N_{0}=2 V-\min \left[2 V,\left(E-E_{0}+F\right)\right]
$$

The minimum function is used in Eq. (C17), because the rank of a matrix cannot exceed its largest dimension.

When $K_{A}>0$, the rank $M$ can take on a continuum of values; however, it is still possible to give bounds for the rank of $M$. First, even when all tensions and pressures vanish, i.e., $\tau_{m}=0, \sigma_{m}=0$ on all edges, the ranks of both $H$ and $\tilde{H}$ still remain at $F$. This result provides the lower bound of $\operatorname{rank}(M) \geq 2 F$. On the other hand, even when all tensions and pressures are finite, the second term in Eq. (C16) cannot exceed $E+F$. We therefore obtain the limits for the constraint counting Eq. (C15):

$$
2 V-(E+F) \leq N_{0} \leq 2 V-2 F .
$$

When zero tension edges are taken into account, we obtain the more general result

$$
N_{0}=\overbrace{2 V}^{\text {d.o.f. }}-\overbrace{\min \left[2 V, \max \left(2 F, E-E_{0}+F\right)\right]}^{\text {no of indep constraints }} .
$$

We explicitly test this in a system of $F=625$ cells at fixed $Z=3$. In Fig. 6 , the number of zero modes is first calculated directed from the Hessian matrix and plotted for states at various values of $p_{0}$. The quantity $2 V-(E-$ $\left.E_{0}+F\right)$ is also plotted as a function of $p_{0}$ for the same states, and they closely track the behavior of $N_{0}$ until $2 \mathrm{~V}-$ $\left(E-E_{0}+F\right)$ becomes larger than the upper limit of zero modes, $2 V-2 F$.

Equation (C18) also allows us to make interesting predictions. Using the fixed topological relations $E=$ $Z V / 2$ and $F=(Z-2) V / 2$, Eq. (C18) becomes a condition for the fraction of zero modes in the system $f_{0}$, which is given by

$$
f_{0} \equiv \frac{N_{0}}{2 V} \in\left[0, \frac{4-Z}{2}\right] .
$$

Equation (C18) suggests that, when $Z>4$, no zero modes should be present in the system. At $Z=3$, the fraction of zero modes is at most $1 / 2$.

In Table I, we list the Maxwell counting for both $K_{A}=0$ and $K_{A}>0$ cases, with the rigidity criterion as well as the number of zero modes in the system for all possible cases.

\section{APPENDIX D: EFFECTIVE MEDIUM THEORY DETAILS}

We derive the effective medium theory for the case with only perimeter constraints, $K_{A}=0$ and $K_{P}>0$. To approximate the scattering behavior of the Hessian $M=K_{P} S^{\|} H S^{\| T}+K_{P} S^{\perp} T S^{\perp T}$, we propose the following effective medium:

$$
\overline{\mathcal{M}}=\mathcal{M}^{\text {topo }}+k_{\text {eff }} \mathcal{M}^{s s},
$$

with $\mathcal{M}^{\text {topo }}$ mapping to the cell topology term $K_{P} S^{\|} H S^{\| T}$ and $k_{\text {eff }} \mathcal{M}^{s s}$ corresponding to the stress contribu-

\begin{tabular}{|c|c|c|c|}
\hline Model & $N_{\text {constraint }}=\operatorname{rank}(M)$ & Rigidity criterion $\left(N_{\text {d.o.f. }}>N_{\text {constraint }}\right)$ & $N_{0}=N_{\text {d.o.f. }}-N_{\text {constraint }^{\mathrm{a}}}$ \\
\hline$K_{A}=0$ & $\min \left[2 V, \max \left(E+F-E_{0}, F\right)\right]$ & $Z \geq\left[3 /\left(1-E_{0} / 2 E\right)\right]$ if $(2-6 / Z) E<E_{0}$ & $N_{0}= \begin{cases}(3-Z) V+E_{0} & \text { if }(2-6 / Z) E<E_{0} \\
0 & \text { otherwise }\end{cases}$ \\
\hline$K_{A}>0$ & $\min \left[2 V, \max \left(E+F-E_{0}, 2 F\right)\right]$ & $\begin{array}{ll}Z \geq\left[3 /\left(1-E_{0} / 2 E\right)\right] & \text { if }(2-6 / Z) E<E_{0}<E / 2 \\
Z \geq 4 & \text { if } E_{0}>E / 2\end{array}$ & $N_{0}= \begin{cases}(3-Z) V+E_{0} & \text { if }(2-6 / Z) E<E_{0}<E / 2 \\
(4-Z) V & \text { if } E_{0}>E / 2 \\
0 & \text { otherwise }\end{cases}$ \\
\hline
\end{tabular}
tion $K_{P} S^{\perp} T S^{\perp T}$.

TABLE I. An overview of constraint counting in the vertex model. The cases for when $K_{A}=0$ and $K_{A}>0$ are presented separately. The rigidity criterion is the minimum value of $Z$ needed to ensure rigidity. Note that the counts of edges $(E)$, vertices $(V)$, and cells $(F)$ are related through the Euler characteristic $(V-E+F=0)$ and the definition for vertex coordination $Z=2 E / V$.

\footnotetext{
${ }^{\mathrm{a}}$ Excluding the $d=2$ number of trivial transitional zero modes.
} 


\section{General stress distribution}

We consider a general distribution of random stresses on edges $k_{m}=\left(\tau_{m} / L_{m}\right)$ :

$$
p(k)=P \rho(k)+(1-P) \delta(k),
$$

where function $\rho(k)$ is normalized in $k>0$ with a single stress scale $\bar{k}$ :

$$
\rho(k)=\frac{1}{\bar{k}} \hat{\rho}\left(\begin{array}{l}
k \\
\overline{\bar{k}}
\end{array}\right)
$$

Following the standard coherent potential approximation procedure [67], the self-consistent equation of the effective medium reads

$P \int_{0}^{\infty} d k \rho(k) \frac{k-k_{\mathrm{eff}}}{1+\left(k-k_{\mathrm{eff}}\right) G_{m}}+(1-P) \frac{-k_{\mathrm{eff}}}{1-k_{\mathrm{eff}} G_{m}}=0$

where $G_{m}=\langle\overline{\mathcal{G}}\rangle_{m}=\sum_{i \mu, j \nu} g_{m i} e_{i \mu}^{\perp} \overline{\mathcal{G}} g_{m j} e_{j \nu}^{\perp}$ is singular as $G_{m}=\left(h / k_{\text {eff }}\right)$ with $h=[(6-Z) / Z]$. Expanding near vanishing stress to the first order of $k_{\text {eff }}$, we get

$$
k_{\mathrm{eff}}=\bar{k} \frac{h}{a P} \frac{P-h}{1-h}, \quad a=\int_{0}^{\infty} d x \frac{1}{x} \hat{\rho}(x) .
$$

\section{2. $G_{m}$ on honeycomb lattice}

We derive $G_{m}$ on a honeycomb lattice, where $Z=3$. The honeycomb lattice contain two particles per unit cell, as shown in Fig. 7, so the displacement is described by a fourdimensional vector:

$$
\mathbf{u}_{l}=\left(u_{l, A, x}, u_{l, A, y}, u_{l, B, x}, u_{l, B, y}\right)
$$

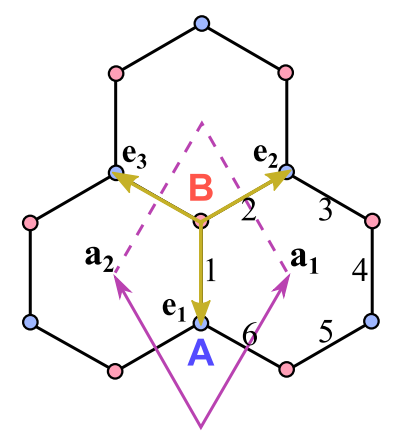

FIG. 7. Primitive cells of the honeycomb lattice.

for each unit cell $l$ at $\mathbf{R}_{l}=\mathbf{R}_{\left(l_{1}, l_{2}\right)}=l_{1} \mathbf{a}_{1}+l_{2} \mathbf{a}_{2}$, where $\mathbf{a}$ are primitive translation vectors. Setting the edge length of hexagons to unity, $\mathbf{a}_{1}=\sqrt{3}\left(\frac{1}{2}, \frac{\sqrt{3}}{2}\right)$ and $\mathbf{a}_{2}=\sqrt{3}\left(-\frac{1}{2}, \frac{\sqrt{3}}{2}\right)$.

In Fourier space,

$$
\begin{aligned}
& \mathbf{u}_{\mathbf{q}}=\sum_{l} \mathbf{u}_{l} e^{-i \mathbf{q} \cdot \mathbf{R}_{l},} \\
& \mathbf{u}_{l}=\frac{1}{N} \sum_{\mathbf{q}} \mathbf{u}_{\mathbf{q}} e^{i \mathbf{q} \cdot \mathbf{R}_{l},}
\end{aligned}
$$

the elastic energy change becomes

$$
\Delta U=\frac{1}{2 N^{2}} \sum_{\mathbf{q}, \mathbf{q}^{\prime}} \mathbf{u}_{\mathbf{q}} \cdot \overline{\mathcal{M}}_{-\mathbf{q}, \mathbf{q}^{\prime}} \cdot \mathbf{u}_{\mathbf{q}^{\prime}},
$$

where $N$ is the number of unit cells. For a homogeneous honeycomb lattice,

$$
\overline{\mathcal{M}}_{\mathbf{q}, \mathbf{q}^{\prime}}=N \delta_{\mathbf{q}, \mathbf{q}^{\prime}} \mathcal{M}_{\mathbf{q}}
$$

We first need to compute the band structure for the first term in the Hessian of the cell perimeter constraints when $k_{\text {eff }}=0$. Each unit cell contains a hexagonal cell on a lattice. The six edges of a cell, labeled in Fig. 7, are

$$
\begin{aligned}
& \mathbf{B}_{1, \mathbf{q}}=(0,1,0,-1), \quad \mathbf{B}_{2, \mathbf{q}}=\left(-\frac{\sqrt{3}}{2} e^{-i\left(\frac{\sqrt{3}}{2} q_{x}+\frac{3}{2} q_{y}\right)},-\frac{1}{2} e^{-i\left(\frac{\sqrt{3}}{2} q_{x}+\frac{3}{2} q_{y}\right)}, \frac{\sqrt{3}}{2}, \frac{1}{2}\right), \\
& \mathbf{B}_{3, \mathbf{q}}=\left(\frac{\sqrt{3}}{2} e^{-i\left(\frac{\sqrt{3}}{2} q_{x}+\frac{3}{2} q_{y}\right)},-\frac{1}{2} e^{-i\left(\frac{\sqrt{3}}{2} q_{x}+\frac{3}{2} q_{y}\right)},-\frac{\sqrt{3}}{2} e^{-i \sqrt{3} q_{x}}, \frac{1}{2} e^{-i \sqrt{3} q_{x}}\right), \quad \mathbf{B}_{4, \mathbf{q}}=\left(0, e^{-i \sqrt{3} q_{x}}, 0,-e^{-i \sqrt{3} q_{x}}\right), \\
& \mathbf{B}_{5, \mathbf{q}}=\left(-\frac{\sqrt{3}}{2} e^{-i \sqrt{3} q_{x}},-\frac{1}{2} e^{-i \sqrt{3} q_{x}}, \frac{\sqrt{3}}{2} e^{-i\left(\frac{\sqrt{3}}{2} q_{x}-\frac{3}{2} q_{y}\right)}, \frac{1}{2} e^{-i\left(\frac{\sqrt{3}}{2} q_{x}-\frac{3}{2} q_{y}\right)}\right), \quad \mathbf{B}_{6, \mathbf{q}}=\left(\frac{\sqrt{3}}{2},-\frac{1}{2},-\frac{\sqrt{3}}{2} e^{-i\left(\frac{\sqrt{3}}{2} q_{x}-\frac{3}{2} q_{y}\right)}, \frac{1}{2} e^{-i\left(\frac{\sqrt{3}}{2} q_{x}-\frac{3}{2} q_{y}\right)}\right) .
\end{aligned}
$$

The corresponding Hessian is

$$
\mathcal{M}_{\mathbf{q}}^{\text {topo }}=\sum_{m, l=1}^{6} \mathbf{B}_{m, \mathbf{q}} \otimes \mathbf{B}_{l,-\mathbf{q}}=\left(\begin{array}{ll}
\mathcal{M}_{1}^{\text {topo }} & \mathcal{M}_{12}^{\text {topo }} \\
\mathcal{M}_{21}^{\text {topo }} & \mathcal{M}_{2}^{\text {topo }}
\end{array}\right),
$$

where 


$$
\begin{gathered}
\mathcal{M}_{1}^{\text {topo }}=\mathcal{M}_{2}^{\text {topo* }}=\left(\begin{array}{cc}
\frac{3}{2}\left(1-\cos \sqrt{3} q_{x}\right) & \sqrt{3} i\left(\frac{1}{2} \sin \sqrt{3} q_{x}-\sin \frac{\sqrt{3}}{2} q_{x} e^{i \frac{3}{2} q_{y}}\right) \\
-\sqrt{3} i\left(\frac{1}{2} \sin \sqrt{3} q_{x}-\sin \frac{\sqrt{3}}{2} q_{x} e^{-i \frac{3}{2} q_{y}}\right) & \frac{3}{2}+\frac{1}{2} \cos \sqrt{3} q_{x}-2 \cos \frac{\sqrt{3}}{2} q_{x} \cos \frac{3}{2} q_{y}
\end{array}\right), \\
\mathcal{M}_{12}^{\text {topo }}=\mathcal{M}_{21}^{\text {topo } *}=\left(\begin{array}{cc}
\frac{3}{2}\left(1-\cos \sqrt{3} q_{x}\right) & -\sqrt{3} i\left(\frac{1}{2} \sin \sqrt{3} q_{x}-\sin \frac{\sqrt{3}}{2} q_{x} e^{-i \frac{3}{2} q_{y}}\right) \\
-\sqrt{3} i\left(\frac{1}{2} \sin \sqrt{3} q_{x}-\sin \frac{\sqrt{3}}{2} q_{x} e^{-i \frac{3}{2} q_{y}}\right) & -\cos ^{2} \frac{\sqrt{3}}{2} q_{x}+2 \cos \frac{\sqrt{3}}{2} q_{x} e^{-i \frac{3}{2} q_{y}}-e^{-i 3 y}
\end{array}\right) .
\end{gathered}
$$

There are three zero bands and one acoustic branch for each unit cell. Besides a normalization prefactor, they are

$\tilde{\psi}_{0,1}=\left(\begin{array}{c}\mathbf{g} \\ e^{-i \mathbf{q} \cdot \hat{\mathbf{e}}_{1}} c(\mathbf{q}) \mathbf{g}^{*}\end{array}\right), \quad \tilde{\psi}_{0,2}=\left(\begin{array}{c}\mathbf{g} \\ -e^{-i \mathbf{q} \cdot \hat{\mathbf{e}}_{1}} c(\mathbf{q}) \mathbf{g}^{*}\end{array}\right), \quad \tilde{\psi}_{0,3}=\left(\begin{array}{c}\mathbf{f} \\ e^{-i \mathbf{q} \cdot \hat{\mathbf{e}}_{1} \mathbf{f}^{*}}\end{array}\right), \quad \tilde{\psi}_{a}=\left(\begin{array}{c}\mathbf{f} \\ -e^{-i \mathbf{q} \cdot \hat{\mathbf{e}}_{1} \mathbf{f}^{*}}\end{array}\right)$,

where $\quad \mathbf{f}=\sum_{j=1}^{3} \hat{\mathbf{e}}_{j} e^{i \mathbf{q} \cdot \hat{\mathbf{e}}_{j}}, \quad \mathbf{g}=\sum_{j=1}^{3} \hat{\mathbf{e}}_{j}^{\perp} e^{-i \mathbf{q} \cdot \hat{\mathbf{e}}_{j}}, \quad$ and $\quad c(\mathbf{q})=-\{1 /[\sqrt{3+2 \eta(\mathbf{q})}]\} \sum_{j=1}^{3} e^{-i \mathbf{q} \cdot \hat{\mathbf{e}}_{j}} \quad$ with $\quad \eta(\mathbf{q})=$ $\frac{1}{2} \sum_{j \neq k} e^{i \mathbf{q} \cdot\left(\hat{\mathbf{e}}_{j}-\hat{\mathbf{e}}_{k}\right)}$. The acoustic branch corresponds to dilation modes of cells, obeying the dispersion relation $\lambda_{a}^{\text {topo }}=2[3-\eta(\mathbf{q})]$.

Include the internal tension $k_{\text {eff }}>0$ contribution to the Hessian matrix,

$$
k_{\mathrm{eff}} \mathcal{M}_{\mathbf{q}}^{s s}=k_{\mathrm{eff}} \sum_{j=1}^{3} \mathbf{D}_{j, \mathbf{q}} \otimes \mathbf{D}_{j,-\mathbf{q}}=k_{\mathrm{eff}}\left(\begin{array}{cc}
\mathcal{M}_{1}^{s s} & \mathcal{M}_{12}^{s s} \\
\mathcal{M}_{21}^{s s} & \mathcal{M}_{2}^{s s}
\end{array}\right),
$$

where vectors $\mathbf{D}$ correspond to the perpendicular directions

$$
\begin{gathered}
\mathbf{D}_{1, \mathbf{q}}=(-1,0,1,0) ; \\
\mathbf{D}_{2, \mathbf{q}}=\left(\frac{1}{2} e^{-i\left(\frac{\sqrt{3}}{2} q_{x}+\frac{3}{2} q_{y}\right)},-\frac{\sqrt{3}}{2} e^{-i\left(\frac{\sqrt{3}}{2} q_{x}+\frac{3}{2} q_{y}\right)},-\frac{1}{2}, \frac{\sqrt{3}}{2}\right) ; \\
\mathbf{D}_{3, \mathbf{q}}=\left(\frac{1}{2} e^{-i\left(-\frac{\sqrt{3}}{2} q_{x}+\frac{3}{2} q_{y}\right)}, \frac{\sqrt{3}}{2} e^{-i\left(-\frac{\sqrt{3}}{2} q_{x}+\frac{3}{2} q_{y}\right)},-\frac{1}{2},-\frac{\sqrt{3}}{2}\right), \\
\mathcal{M}_{1}^{s s}=\mathcal{M}_{2}^{s s}=\frac{3}{2} \mathcal{I}, \\
\mathcal{M}_{12}^{s s}=\mathcal{M}_{21}^{s s *}=\left(\begin{array}{cc}
-1-\frac{1}{2} e^{-i \frac{3}{2} q_{y}} \cos \frac{\sqrt{3}}{2} q_{x} & -i \frac{\sqrt{3}}{2} e^{-i \frac{3}{2} q_{y}} \sin \frac{\sqrt{3}}{2} q_{x} \\
-i \frac{\sqrt{3}}{2} e^{-i \frac{3}{2} q_{y}} \sin \frac{\sqrt{3}}{2} q_{x} & -\frac{3}{2} e^{-i \frac{3}{2} q_{y}} \cos \frac{\sqrt{3}}{2} q_{x}
\end{array}\right) .
\end{gathered}
$$

The band structure of $\mathcal{M}^{s s}$ includes one zero band, one acoustic branch, and two optical branches:

$$
\tilde{\phi}_{0}=\left(\begin{array}{c}
\mathbf{f} \\
-e^{-i \mathbf{q} \cdot \hat{\mathbf{e}}_{1} \mathbf{f}^{*}}
\end{array}\right), \quad \tilde{\phi}_{a}=\left(\begin{array}{c}
\mathbf{g} \\
e^{-i \mathbf{q} \cdot \hat{\mathbf{e}}_{1}} c(\mathbf{q}) \mathbf{g}^{*}
\end{array}\right), \quad \tilde{\phi}_{o, 1}=\left(\begin{array}{c}
\mathbf{g} \\
-e^{-i \mathbf{q} \cdot \hat{\mathbf{e}}_{1}} c(\mathbf{q}) \mathbf{g}^{*}
\end{array}\right), \quad \tilde{\phi}_{o, 2}=\left(\begin{array}{c}
\mathbf{f} \\
e^{-i \mathbf{q} \cdot \hat{\mathbf{e}}_{1} \mathbf{f}^{*}}
\end{array}\right)
$$

corresponding to $\lambda_{0}^{s s}=0, \lambda_{a}^{s s}=\frac{3}{2}-\{[\sqrt{3+2 \eta(\mathbf{q})}] / 2\}, \lambda_{o, 1}^{s s}=\frac{3}{2}+\{[\sqrt{3+2 \eta(\mathbf{q})}] / 2\}$, and $\lambda_{o, 2}^{s s}=3$.

Notice that the only acoustic branch of $\mathcal{M}_{\mathbf{q}}^{\text {topo }}$ is the only zero band of $\mathcal{M}_{\mathbf{q}}^{s s}$. So when there is internal tension $k_{\text {eff }}>0$, the full Hessian matrix

$$
\overline{\mathcal{M}}_{\mathbf{q}}\left(k_{\text {eff }}\right)=\mathcal{M}_{\mathbf{q}}^{\text {topo }}+k_{\text {eff }} \mathcal{M}_{\mathbf{q}}^{s s}
$$

is invertible: 


$$
G_{m}=\int_{1 B Z} \frac{d^{2} \mathbf{q}}{v_{1}} \mathbf{D}_{1,-\mathbf{q}} \cdot \mathcal{U}_{\mathbf{q}} \mathcal{U}_{\mathbf{q}}^{\dagger}\left[\overline{\mathcal{M}}_{\mathbf{q}}\left(k_{\mathrm{eff}}\right)-\omega^{2} \mathcal{I}\right]^{-1} \mathcal{U}_{\mathbf{q}} \mathcal{U}_{\mathbf{q}}^{\dagger} \cdot \mathbf{D}_{1, \mathbf{q}}
$$

where

$$
\begin{aligned}
\mathcal{U}_{\mathbf{q}} & =\left(\phi_{0}, \phi_{a}, \phi_{o, 1}, \phi_{o, 2}\right) \\
& =\frac{1}{2 \sqrt{3-\eta(\mathbf{q})}}\left(\begin{array}{cccc}
\mathbf{f} & \mathbf{g} & \mathbf{g} & \mathbf{f} \\
-e^{-i \mathbf{q} \cdot \hat{\mathbf{e}}_{1}} \mathbf{f}^{*} & e^{-i \mathbf{q} \cdot \hat{\mathbf{e}}_{1}} c(\mathbf{q}) \mathbf{g}^{*} & -e^{-i \mathbf{q} \cdot \hat{\mathbf{e}}_{1}} c(\mathbf{q}) \mathbf{g}^{*} & e^{-i \mathbf{q} \cdot \hat{\mathbf{e}}_{1} \mathbf{f}^{*}}
\end{array}\right) .
\end{aligned}
$$

So $\left.G_{m}\right|_{\omega=0}=\left(1 / k_{\mathrm{eff}}\right)$.

\section{Phase diagram far from $Z=3$}

In the main text, we derive the phase diagram near the critical point $\left(Z=3, p_{0}^{*}\right)$, illustrated by the left panel in Fig. 8. Though we do see a crossover from the perimeterdominated region to the topology-dominated region, the nature of the rigidity does not change in the vicinity of trijunction tissues $Z=3$. As long as the number of constraints $2 F<2 V$ and $Z<Z_{c}=4$ (or when $K_{A}=0$, $F<2 V$ and $Z<Z_{c}=6$ ), internal tension $k>0$ is
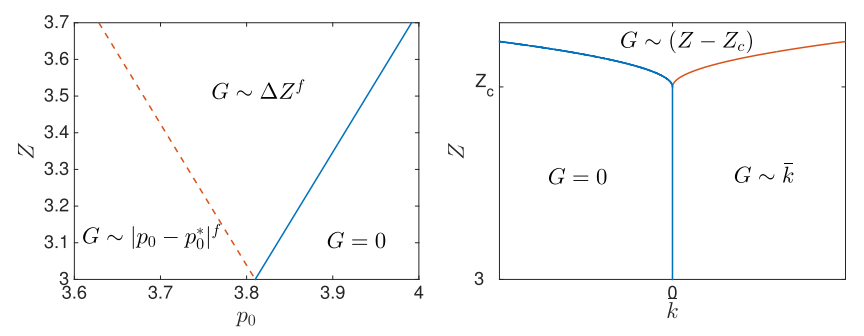

FIG. 8. Left: Phase diagram $Z-p_{0}$. Right: Phase diagram $Z-k$, where $Z_{c}=4$ for $K_{A}>0$ and $Z_{c}=6$ for $K_{A}=0$. The solid-fluid boundary is shown by the blue line. The red lines separate the regions where mechanical moduli are dominated by different control parameters.

(a)

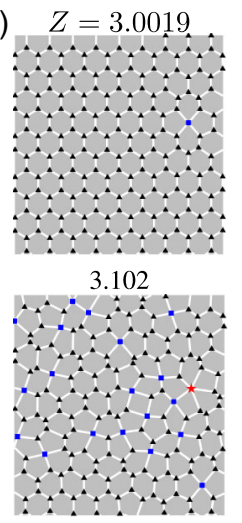

3.0588

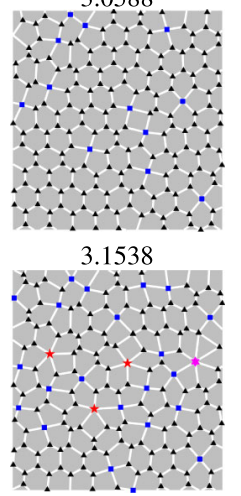

necessary to stabilize the structure, as illustrated in the phase diagram with internal tension as a control parameter in the right panel in Fig. 8. Above $Z_{c}$, the topology alone can rigidify the structure, and the solid-liquid transition happens along the line $Z-Z_{c} \sim \sqrt{-k}$ as predicted in Ref. [66], shown in Fig. 8. In this new topology-dominated region, the shear modulus crosses over to a linear dependence on the junction connectivity $Z-Z_{c}$.
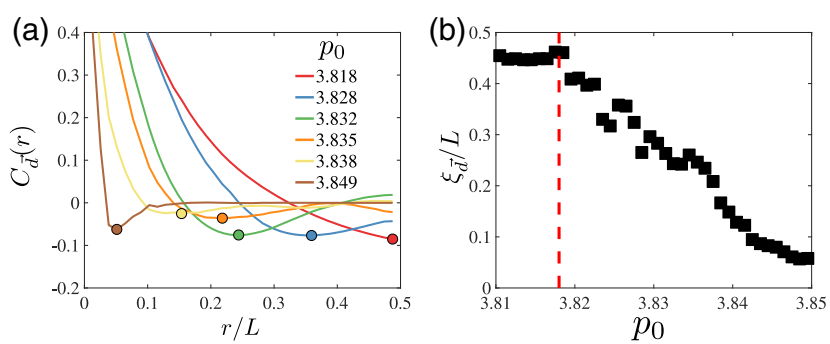

FIG. 10. (a) Spatial autocorrelation of the vertex displacement field after a single edge is pinched at $Z=3$. The dot on each curve indicates the location of the negative dip in $C_{\vec{d}}(r)$ which defines the swirl size $\xi_{d}$. (b) The swirl size $\xi_{d}$ associated with a single edge pinch at $Z=3$. In the rigid tissue, the swirl size spans on the order of the tissue size but decreases in the fluid tissue. This result suggests that, near the fluid-solid transition, a single pinched edge has long-range effects, whereas deeper in the fluid state the effect of pinching is much more local.

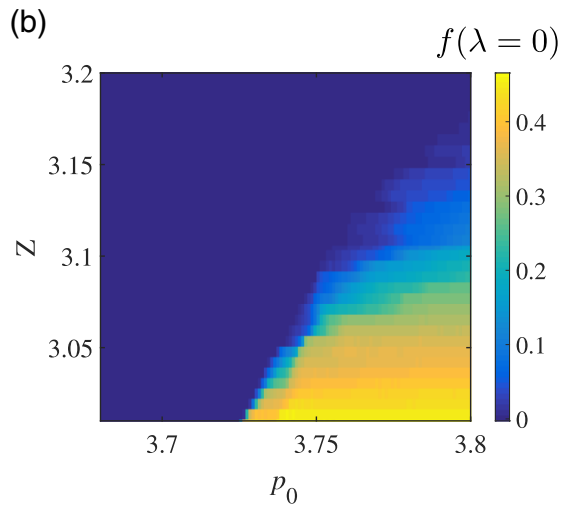

FIG. 9. (a) Application of the edge pinch to an initially ordered hexagonal lattice. (b) The corresponding $Z-p_{0}$ phase diagram. The color bar gives the fraction of floppy modes in the tissue. 


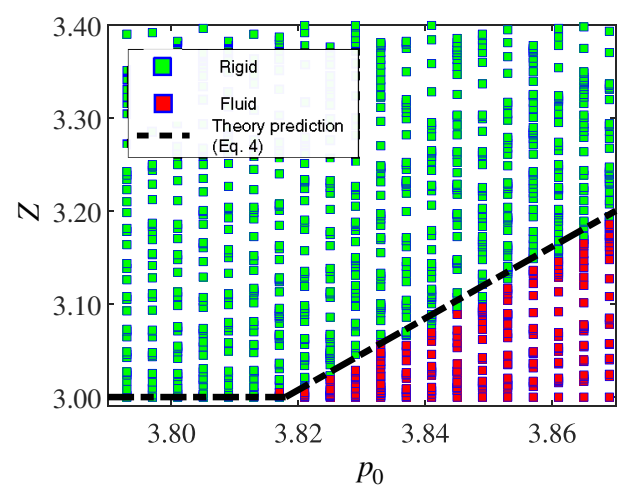

FIG. 11. We also perform an alternative set of simulations in which $T 1$ and $T 2$ (cell apoptosis) transitions are allowed. Specifically, $T 1$ transitions are attempted on an edge when its length is shorter than $16 \%$ of the average edge lengths in the system, while $T 2$ transitions are carried out on cells with an area less than $10 \%$ of the average cell area. The phase diagram for tissue mechanical states remains the same with this protocol.

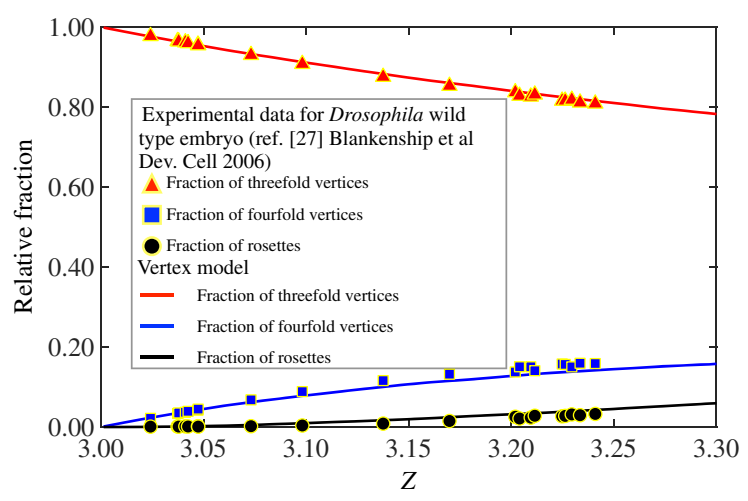

FIG. 12. A direct comparison between the topologies generated in the vertex model and experimental observations in a wild-type Drosophila embryo [33]. The relative fractions for (i) threefold vertices, (ii) fourfold vertices, and (iii) rosettes are plotted as a function of $Z$.

[1] T. Nagai and H. Honda, A Dynamic Cell Model for the Formation of Epithelial Tissues, Philos. Mag. B 81, 699 (2001).

[2] R. Farhadifar, J.-C. Roeper, B. Aigouy, S. Eaton, and F. Julicher, The Influence of Cell Mechanics, Cell-Cell Interactions, and Proliferation on Epithelial Packing, Curr. Biol. 17, 2095 (2007).

[3] L. Hufnagel, A. A. Teleman, H. Rouault, S. M. Cohen, and B. I. Shraiman, On the Mechanism of Wing Size Determination in Fly Development, Proc. Natl. Acad. Sci. U.S.A. 104, 3835 (2007).

[4] D. B. Staple, R. Farhadifar, J. C. Roeper, B. Aigouy, S. Eaton, and F. Julicher, Mechanics and Remodelling of Cell Packings in Epithelia, Eur. Phys. J. E 33, 117 (2010).
[5] M. L. Manning, R. A. Foty, M. S. Steinberg, and E.-M. Schoetz, Coaction of Intercellular Adhesion and Cortical Tension Specifies Tissue Surface Tension, Proc. Natl. Acad. Sci. U.S.A. 107, 12517 (2010).

[6] A. G. Fletcher, M. Osterfield, R. E. Baker, and S. Y. Shvartsman, Vertex Models of Epithelial Morphogenesis, Biophys. J. 106, 2291 (2014).

[7] D. Bi, J. H. Lopez, J. M. Schwarz, and M. L. Manning, Energy Barriers and Cell Migration in Densely Packed Tissues, Soft Matter 10, 1885 (2014).

[8] D. Bi, J. H. Lopez, J. M. Schwarz, and M. L. Manning, A Density-Independent Rigidity Transition in Biological Tissues, Nat. Phys. 11, 1074 (2015).

[9] D. Bi, X. Yang, M. C. Marchetti, and M. L. Manning, Motility-Driven Glass and Jamming Transitions in Biological Tissues, Phys. Rev. X 6, 021011 (2016).

[10] X. Yang, D. Bi, M. Czajkowski, M. Merkel, M. L. Manning, and M. C. Marchetti, Correlating Cell Shape and Cellular Stress in Motile Confluent Tissues, Proc. Natl. Acad. Sci. U.S.A. 114, 12663 (2017).

[11] M. Merkel and M. L. Manning, A Geometrically Controlled Rigidity Transition in a Model for Confluent $3 d$ Tissues, New J. Phys. 20, 022002 (2018).

[12] N. Noll, M. Mani, I. Heemskerk, S. J. Streichan, and B. I. Shraiman, Active Tension Network Model Suggests an Exotic Mechanical State Realized in Epithelial Tissues, Nat. Phys. 13, 1221 (2017).

[13] A. Boromand, A. Signoriello, F. Ye, C. S. O'Hern, and M. D. Shattuck, Jamming of Deformable Polygons, Phys. Rev. Lett. 121, 248003 (2018).

[14] E. Teomy, D. A. Kessler, and H. Levine, Confluent and Nonconfluent Phases in a Model of Cell Tissue, Phys. Rev. E 98, 042418 (2018).

[15] D. M. Sussman, CellGPU: Massively Parallel Simulations of Dynamic Vertex Models, Comput. Phys. Commun. 219, 400 (2017).

[16] M. A. Spencer, Z. Jabeen, and D. K. Lubensky, Vertex Stability and Topological Transitions in Vertex Models of Foams and Epithelia, Eur. Phys. J. E 40, 2 (2017).

[17] D. L. Barton, S. Henkes, C. J. Weijer, and R. Sknepnek, Active Vertex Model for Cell-Resolution Description of Epithelial Tissue Mechanics, PLoS Comput. Biol. 13, e1005569 (2017).

[18] M. Moshe, M. J. Bowick, and M. C. Marchetti, Geometric Frustration and Solid-Solid Transitions in Model 2d Tissue, Phys. Rev. Lett. 120, 268105 (2018).

[19] L. Atia, D. Bi, Y. Sharma, J. A. Mitchel, B. Gweon, S. A. Koehler, S. J. DeCamp, B. Lan, J. H. Kim, R. Hirsch, A. F. Pegoraro, K. H. Lee, J. R. Starr, D. A. Weitz, A. C. Martin, J.-A. Park, J. P. Butler, and J J. Fredberg, Geometric Constraints during Epithelial Jamming, Nat. Phys. 14, 613 (2018).

[20] S. Alt, P. Ganguly, and G. Salbreux, Vertex Models: From Cell Mechanics to Tissue Morphogenesis, Phil. Trans. R. Soc. B 372, 20150520 (2017).

[21] F. Giavazzi, M. Paoluzzi, M. Macchi, D. Bi, G. Scita, M. L. Manning, R. Cerbino, and M. C. Marchetti, Flocking Transitions in Confluent Tissues, Soft Matter 14, 3471 (2018). 
[22] M. Czajkowski, D. Bi, M. L. Manning, and M. C. Marchetti, Hydrodynamics of Shape-Driven Rigidity Transitions in Motile Tissues, Soft Matter 14, 5628 (2018).

[23] M. F. Staddon, D. Bi, A. P. Tabatabai, V. Ajeti, M. P. Murrell, and S. Banerjee, Cooperation of Dual Modes of Cell Motility Promotes Epithelial Stress Relaxation to Accelerate Wound Healing, PLoS Comput. Biol. 14, e1006502 (2018).

[24] X. Li, A. Das, and D. Bi, Biological Tissue-Inspired Tunable Photonic Fluid, Proc. Natl. Acad. Sci. U.S.A. 115, 6650 (2018).

[25] J. A. Parket al., Unjamming and Cell Shape in the Asthmatic Airway Epithelium, Nat. Mater. 14, 1040 (2015).

[26] M. J. Harding, H. F. McGraw, and A. Nechiporuk, The Roles and Regulation of Multicellular Rosette Structures during Morphogenesis, Development 141, 2549 (2014).

[27] D. Weaire and N. Rivier, Soap, Cells and StatisticsRandom Patterns in Two Dimensions, Contemp. Phys. 25, 59 (1984).

[28] J. A. Zallen and E. Wieschaus, Patterned Gene Expression Directs Bipolar Planar Polarity in Drosophila, Dev. Cell 6, 343 (2004).

[29] C. Bertet, L. Sulak, and T. Lecuit, Myosin-Dependent Junction Remodelling Controls Planar Cell Intercalation and Axis Elongation, Nature (London) 429, 667 (2004).

[30] J. A. Zallen and R. Zallen, Cell-Pattern Disordering during Convergent Extension in Drosophila, J. Phys. Condens. Matter 16, S5073 (2004).

[31] M. Rauzi, P. Verant, T. Lecuit, and P.-F. Lenne, Nature and Anisotropy of Cortical Forces Orienting Drosophila Tissue Morphogenesis, Nat. Cell Biol. 10, 1401 (2008).

[32] L. M. Escudero, M. Bischoff, and M. Freeman, Myosin II Regulates Complex Cellular Arrangement and Epithelial Architecture in Drosophila, Dev. Cell 13, 717 (2007).

[33] J. T. Blankenship, S. T. Backovic, J. S. P. Sanny, O. Weitz, and J.A. Zallen, Multicellular Rosette Formation Links Planar Cell Polarity to Tissue Morphogenesis., Dev. Cell 11, 459 (2006).

[34] R. Fernandez-Gonzalez, S. de Matos Simoes, J.-C. Röper, S. Eaton, and J. A. Zallen, Myosin II Dynamics Are Regulated by Tension in Intercalating Cells, Dev. Cell 17, 736 (2009).

[35] M. Tamada, D. L. Farrell, and J. A. Zallen, Abl Regulates Planar Polarized Junctional Dynamics through $\beta$-Catenin Tyrosine Phosphorylation, Dev. Cell 22, 309 (2012).

[36] K. E. Kasza, D. L. Farrell, and J. A. Zallen, Spatiotemporal Control of Epithelial Remodeling by Regulated Myosin Phosphorylation, Proc. Natl. Acad. Sci. U.S.A. 111, 11732 (2014).

[37] Z. Sun, C. Amourda, M. Shagirov, Y. Hara, T. E. Saunders, and Y. Toyama, Basolateral Protrusion and Apical Contraction Cooperatively Drive Drosophila Germ-Band Extension, Nat. Cell Biol. 19, 375 (2017).

[38] F. Robertson, N. Pinal, P. Fichelson, and F. Pichaud, Atonal and EGFR Signalling Orchestrate Rok- and DrakDependent Adherens Junction Remodelling during Ommatidia Morphogenesis, Development 139, 3432 (2012).

[39] N. Gompel, N. Cubedo, C. Thisse, B. Thisse, C. DamblyChaudière, and A. Ghysen, Pattern Formation in the Lateral Line of Zebrafish, Mech. Dev. 105, 69 (2001).
[40] A. Nechiporuk and D W. Raible, FGF-Dependent Mechanosensory Organ Patterning in Zebrafish, Science 320, 1774 (2008).

[41] V. Lecaudey, G. Cakan-Akdogan, W. H. J. Norton, and D. Gilmour, Dynamic Fgf Signaling Couples Morphogenesis and Migration in the Zebrafish Lateral Line Primordium, Development 135, 2695 (2008).

[42] T. Nishimura and M. Takeichi, Shroom3-Mediated Recruitment of Rho Kinases to the Apical Cell Junctions Regulates Epithelial and Neuroepithelial Planar Remodeling, Development 135, 1493 (2008).

[43] M. Williams, W. Yen, X. Lu, and A. Sutherland, Distinct Apical and Basolateral Mechanisms Drive Planar Cell Polarity-Dependent Convergent Extension of the Mouse Neural Plate, Dev. Cell 29, 34 (2014).

[44] G. Trichas, A. M. Smith, N. White, V. Wilkins, T. Watanabe, A. Moore, B. Joyce, J. Sugnaseelan, T. A. Rodriguez, D. Kay, R. E. Baker, P. K. Maini, and S. Srinivas, MultiCellular Rosettes in the Mouse Visceral Endoderm Facilitate the Ordered Migration of Anterior Visceral Endoderm Cells, PLoS Biol. 10, e1001256 (2012).

[45] S. S. Lienkamp, K. Liu, C. M. Karner, T. J. Carroll, O. Ronneberger, J. B. Wallingford, and G. Walz, Vertebrate Kidney Tubules Elongate Using a Planar Cell PolarityDependent, Rosette-Based Mechanism of Convergent Extension, Nat. Genet. 44, 1382 (2012).

[46] A. Villasenor, D. C. Chong, M. Henkemeyer, and O. Cleaver, Epithelial Dynamics of Pancreatic Branching Morphogenesis, Development 137, 4295 (2010).

[47] S.-C. Zhang, M. Wernig, I. D. Duncan, O. Brüstle, and J. A. Thomson, In Vitro Differentiation of Transplantable Neural Precursors from Human Embryonic Stem Cells, Nat. Biotechnol. 19, 1129 (2001).

[48] Y. Elkabetz and L. Studer, Human ESC-Derived Neural Rosettes and Neural Stem Cell Progression, Cold Spring Harbor Symp., Quantitative structure-activity relationships in pharmacology, chemistry, and biology 73, 377 (2008).

[49] F. J. Wippold and A. Perry, Neuropathology for the Neuroradiologist: Rosettes and Pseudorosettes, Am. J. Neuroradiol. 27, 488 (2006).

[50] N. C. Heer and A.C. Martin, Tension, Contraction and Tissue Morphogenesis, Development 144, 4249 (2017).

[51] L. Kocgozlu, T. B. Saw, A. P. Le, I. Yow, M. Shagirov, E. Wong, R. M. Mège, C. T. Lim, Y. Toyama, and B. Ladoux, Epithelial Cell Packing Induces Distinct Modes of Cell Extrusions, Curr. Biol. 26, 2942 (2016).

[52] Y. Toyama, X. G. Peralta, A. R. Wells, D. P. Kiehart, and G. S. Edwards, Apoptotic Force and Tissue Dynamics during Drosophila Embryogenesis, Science 321, 1683 (2008).

[53] E. Marinari, A. Mehonic, S. Curran, J. Gale, T. Duke, and B. Baum, Live-Cell Delamination Counterbalances Epithelial Growth to Limit Tissue Overcrowding, Nature (London) 484, 542 (2012).

[54] G. Slattum, K. M. McGee, and J. Rosenblatt, P115 RhoGEF and Microtubules Decide the Direction Apoptotic Cells Extrude from an Epithelium, J. Cell Biol. 186, 693 (2009).

[55] W. Razzell, W. Wood, and P. Martin, Recapitulation of Morphogenetic Cell Shape Changes Enables Wound ReEpithelialisation, Development 141, 1814 (2014). 
[56] A. Brugués, E. Anon, V. Conte, J. H. Veldhuis, M. Gupta, J. Colombelli, J. J. Muñoz, G. W. Brodland, B. Ladoux, and X. Trepat, Forces Driving Epithelial Wound Healing, Nat. Phys. 10, 683 (2014).

[57] S. J. Streichan, M. F. Lefebvre, N. Noll, E. F. Wieschaus, and B.I. Shraiman, Global Morphogenetic Flow Is Accurately Predicted by the Spatial Distribution of Myosin Motors, eLife 7, e27454 (2018).

[58] B. A. Shook, D. H. Manz, J. J. Peters, S. Kang, and J. C. Conover, Spatiotemporal Changes to the Subventricular Zone Stem Cell Pool through Aging, J. Neurosci. 32, 6947 (2012).

[59] W. Razzell, M. E. Bustillo, and J. A. Zallen, The ForceSensitive Protein Ajuba Regulates Cell Adhesion during Epithelial Morphogenesis, J. Cell Biol. 217, 3715 (2018).

[60] J. C. Maxwell F. R. S., L. on the Calculation of the Equilibrium and Stiffness of Frames, Philos. Mag. 27, 294 (1864).

[61] C.L. Kane and T.C. Lubensky, Topological Boundary Modes in Isostatic Lattices, Nat. Phys. 10, 39 (2014).

[62] A. J. Liu and S. R. Nagel, The Jamming Transition and the Marginally Jammed Solid, Annu. Rev. Condens. Matter Phys. 1, 347 (2010).

[63] S. Feng, M. F. Thorpe, and E. Garboczi, Effective-Medium Theory of Percolation on Central-Force Elastic Networks, Phys. Rev. B 31, 276 (1985).

[64] X. Mao, N. Xu, and T. C. Lubensky, Soft Modes and Elasticity of Nearly Isostatic Lattices: Randomness and Dissipation, Phys. Rev. Lett. 104, 085504 (2010).

[65] M. Wyart, Scaling of Phononic Transport with Connectivity in Amorphous Solids, Europhys. Lett. 89, 64001 (2010).

[66] E. DeGiuli, A. Laversanne-Finot, G. A. Düring, E. Lerner, and M. Wyart, Effects of Coordination and Pressure on Sound Attenuation, Boson Peak and Elasticity in Amorphous Solids, Soft Matter 10, 5628 (2014).

[67] T. C. Lubensky, C. L. Kane, X. Mao, A. Souslov, and K. Sun, Phonons and Elasticity in Critically Coordinated Lattices, Rep. Prog. Phys. 78, 073901 (2015).

[68] C. P. Broedersz, X. Mao, T. C. Lubensky, and F. C. MacKintosh, Criticality and Isostaticity in Fibre Networks, Nat. Phys. 7, 983 (2011).

[69] M. Das, D. A. Quint, and J. M. Schwarz, Redundancy and Cooperativity in the Mechanics of Compositely Crosslinked Filamentous Networks, PLoS One 7, e35939 (2012).

[70] E. M. Lifshitz, A. M. Kosevich, and L. P. Pitaevskii, Chapter I - Fundamental Equations, Theory of Elasticity (Butterworth-Heinemann, Oxford, 1986), p. 109.

[71] M. S. Hutson, Y. Tokutake, M.-S. Chang, J. W. Bloor, S. Venakides, D. P. Kiehart, and G. S. Edwards, Forces for Morphogenesis Investigated with Laser Microsurgery and Quantitative Modeling, Science 300, 145 (2003).

[72] G. W. Brodland, V. Conte, P. G. Cranston, J. Veldhuis, S. Narasimhan, M. S. Hutson, A. Jacinto, F. Ulrich, B. Baum, and M. Miodownik, Video Force Microscopy Reveals the Mechanics of Ventral Furrow Invagination in Drosophila, Proc. Natl. Acad. Sci. U.S.A. 107, 22111 (2010).
[73] K. K. Chiou, L. Hufnagel, and B. I. Shraiman, Mechanical Stress Inference for Two Dimensional Cell Arrays, PLoS Comput. Biol. 8, e1002512 (2012).

[74] S. Ishihara, K. Sugimura, S. J. Cox, I. Bonnet, Y. Bella1che, and F. Graner, Comparative Study of Non-Invasive Force and Stress Inference Methods in Tissue, Eur. Phys. J. E 36, 45 (2013).

[75] S. Ishihara and K. Sugimura, Bayesian Inference of Force Dynamics during Morphogenesis, J. Theor. Biol. 313, 201 (2012).

[76] G. W. Brodland, J. H. Veldhuis, S. Kim, M. Perrone, D. Mashburn, and M.S. Hutson, Cellfit: A Cellular ForceInference Toolkit Using Curvilinear Cell Boundaries, PLoS One 9, e99116 (2014).

[77] D. Bi and L. Yan (private communication).

[78] P.-A. Pouille, P. Ahmadi, A.-C. Brunet, and E. Farge, Mechanical Signals Trigger Myosin II Redistribution and Mesoderm Invagination in Drosophila Embryos, Sci. Signal. (Online) 2, ra16 (2009).

[79] S. Sun and K. D. Irvine, Cellular Organization and Cytoskeletal Regulation of the Hippo Signaling Network, Trends Cell Biol. 26, 694 (2016).

[80] C. Ibar, E. Kirichenko, B. Keepers, E. Enners, K. Fleisch, and K. D. Irvine, Tension-Dependent Regulation of Mammalian Hippo Signaling through LIMD1, J. Cell Sci. 131, jes214700 (2018).

[81] K. Doubrovinski, M. Swan, O. Polyakov, and E. F. Wieschaus, Measurement of Cortical Elasticity in Drosophila Melanogaster Embryos Using Ferrofluids, Proc. Natl. Acad. Sci. U.S.A. 114, 1051 (2017).

[82] M. Merkel, K. Baumgarten, B. P. Tighe, and M. L. Manning, A Unifying Perspective on Rigidity in Under-Constrained Materials, arXiv:1809.01586.

[83] A. Sharma, A. J. Licup, K. A. Jansen, R. Rens, M. Sheinman, G. H. Koenderink, and F. C. MacKintosh, Strain-Controlled Criticality Governs the Nonlinear Mechanics of Fibre Networks, Nat. Phys. 12, 584 (2016).

[84] J. Feng, H. Levine, X. Mao, and L. M. Sander, Alignment and Nonlinear Elasticity in Biopolymer Gels, Phys. Rev. E 91, 042710 (2015).

[85] J. Feng, H. Levine, X. Mao, and L. M. Sander, Nonlinear Elasticity of Disordered Fiber Networks, Soft Matter 12, 1419 (2016).

[86] B. Li and S. X. Sun, Coherent Motions in Confluent Cell Monolayer Sheets, Biophys. J. 107, 1532 (2014).

[87] D. M. Sussman and M. Merkel, No Unjamming Transition in a Voronoi Model of Biological Tissue, Soft Matter 14, 3397 (2018).

[88] R. H. Byrd, P. Lu, J. Nocedal, and C. Zhu, A Limited Memory Algorithm for Bound Constrained Optimization, SIAM J. Sci. Comput. 16, 1190 (1995).

[89] C. E. Maloney and A. Lemaitre, Amorphous Systems in Athermal, Quasistatic Shear, Phys. Rev. E 74, 016118 (2006).

[90] $\operatorname{rank}(A B)=\min [\operatorname{rank}(A), \operatorname{rank}(B)]$.

[91] L. Guttman, Enlargement Methods for Computing the Inverse Matrix, Ann. Math. Stat. 17, 336 (1946). 